\title{
AIP
}

\section{Quantum effects in energy and charge transfer in an artificial photosynthetic complex}

Pulak Kumar Ghosh, Anatoly Yu. Smirnov, and Franco Nori

Citation: J. Chem. Phys. 134, 244103 (2011); doi: 10.1063/1.3600341

View online: http://dx.doi.org/10.1063/1.3600341

View Table of Contents: http://jcp.aip.org/resource/1/JCPSA6/v134/i24

Published by the AIP Publishing LLC.

\section{Additional information on J. Chem. Phys.}

Journal Homepage: http://jcp.aip.org/

Journal Information: http://jcp.aip.org/about/about_the_journal

Top downloads: http://jcp.aip.org/features/most_downloaded

Information for Authors: http://jcp.aip.org/authors

\section{ADVERTISEMENT}

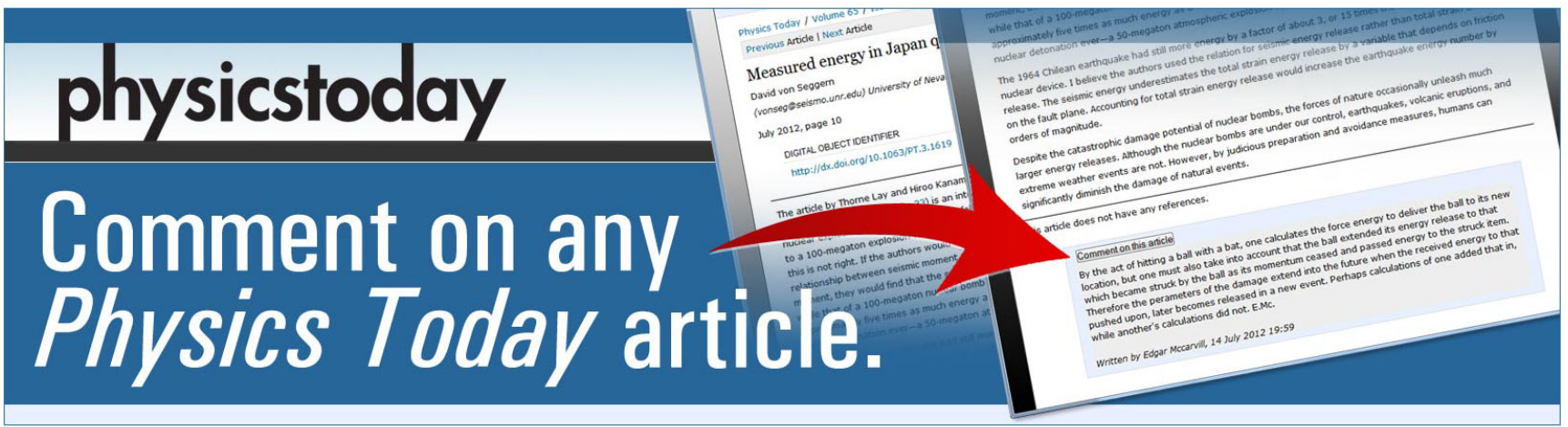




\title{
Quantum effects in energy and charge transfer in an artificial photosynthetic complex
}

\author{
Pulak Kumar Ghosh, ${ }^{1, a)}$ Anatoly Yu. Smirnov, ${ }^{1,2}$ and Franco Nori ${ }^{1,2}$ \\ ${ }^{1}$ Advanced Science Institute, RIKEN, Wako, Saitama 351-0198, Japan \\ ${ }^{2}$ Physics Department, The University of Michigan, Ann Arbor, Michigan 41109-1040, USA
}

(Received 27 January 2011; accepted 25 May 2011; published online 23 June 2011)

\begin{abstract}
We investigate the quantum dynamics of energy and charge transfer in a wheel-shaped artificial photosynthetic antenna-reaction center complex. This complex consists of six light-harvesting chromophores and an electron-acceptor fullerene. To describe quantum effects on a femtosecond time scale, we derive the set of exact non-Markovian equations for the Heisenberg operators of this photosynthetic complex in contact with a Gaussian heat bath. With these equations we can analyze the regime of strong system-bath interactions, where reorganization energies are of the order of the intersite exciton couplings. We show that the energy of the initially excited antenna chromophores is efficiently funneled to the porphyrin-fullerene reaction center, where a charge-separated state is set up in a few picoseconds, with a quantum yield of the order of $95 \%$. In the single-exciton regime, with one antenna chromophore being initially excited, we observe quantum beatings of energy between two resonant antenna chromophores with a decoherence time of $\sim 100 \mathrm{fs}$. We also analyze the double-exciton regime, when two porphyrin molecules involved in the reaction center are initially excited. In this regime we obtain pronounced quantum oscillations of the charge on the fullerene molecule with a decoherence time of about 20 fs (at liquid nitrogen temperatures). These results show a way to directly detect quantum effects in artificial photosynthetic systems. () 2011 American Institute of Physics. [doi:10.1063/1.3600341]
\end{abstract}

\section{INTRODUCTION}

The multistep energy-transduction process in natural photosystems begins with capturing sunlight photons by light-absorbing antenna chromophores surrounding a reaction center. ${ }^{1,2}$ The antenna chromophores transfer radiation energy to the reaction center directly or through a series of accessory chromophores. The reaction center harnesses the excitation energy to create a stable charge-separated state.

Energy transfer in natural and artificial photosynthetic structures has been an intriguing issue in quantum biophysics due to the conspicuous presence of long-lived quantum coherence observed with two-dimensional Fourier transform electronic spectroscopy. ${ }^{3,4}$ These experimental achievements have motivated researchers to investigate the role of quantum coherence in very efficient energy transmission, which takes place in natural photosystems. ${ }^{5-9}$ Quantum coherent effects surviving up to room temperatures have also been observed in artificial polymers. ${ }^{10}$ Artificial photosynthetic elements, mimicking natural photosystems, might serve as building blocks for efficient and powerful sources of energy. ${ }^{11,12}$ Some of these elements have been created and studied experimentally in Refs. 13-18. The theoretical modelling of artificial reaction centers has been recently performed in Refs. 19 and 20.

Here, we study energy transfer and charge separation in a wheel-shaped molecular complex (BPF complex, see Fig. 1) mimicking a natural photosynthetic system. This complex has

\footnotetext{
a) Author to whom correspondence should be addressed. Electronic mail: pulak@ riken.jp.
}

been synthesized and experimentally investigated in Ref. 17. It has four antennas - two bis(phenylethynyl)anthracene (BPEA) molecules and two borondipyrromethene (BDPY) chromophores, as well as two zinc porphyrins $\left(\mathrm{ZnPy}_{a}\right.$ and $\mathrm{ZnPy}_{b}$ ). These six light-absorbing chromophores are attached to a central hexaphenylbenzene core. Electrons can tunnel from the zinc porphyrin molecules to a fullerene $\mathrm{F}$ (electron acceptor). Thus, two porphyrins and the fullerene molecule form an artificial reaction center $\left(\mathrm{ZnPy}_{a}-\mathrm{F}-\mathrm{ZnPy}_{b}\right)$. The BPEA chromophores strongly absorb around $450 \mathrm{~nm}$ (the blue region), while the BDPY moieties have good absorptions around $513 \mathrm{~nm}$ (green region). Porphyrins have absorption peaks at both red and orange wavelengths. Therefore, the BPF complex can utilize most of the rainbow of sunlight - from blue to red photons. It is shown in Ref. 17 that the absorption of photons results in the formation of a porphyrin-fullerene charge-separated state with a lifetime of 230 ps; in doing so, excitations from the BPEA and BDPY antenna chromophores are transferred to the porphyrins with a subsequent donation of an electron from the excited states of the porphyrins to the fullerene moiety. This process takes a few picoseconds, suggesting that the excitonic coupling between chromophores is sufficiently strong. The electronic coupling between the porphyrins and the fullerene controlling tunneling of electrons in the artificial reaction center also should be quite strong. It should be noted, however, that spectroscopic data ${ }^{15-17}$ show that the absorption spectrum of the BPF complex is approximately represented as a superposition of contributions from the individual chromophores with almost no perturbations due to the links between the chromophores. This means that the chromophores comprising the light-harvesting complex can 


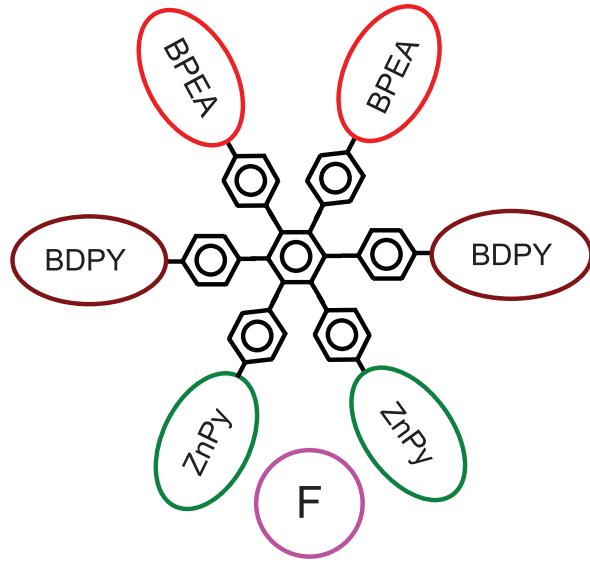

FIG. 1. Schematic diagram of the wheel-shaped artificial antenna-reaction center complex reported in Ref. 17. We use the short notation, BPF complex, to denote this photosynthetic device. The antenna-reaction center complex contains six light-harvesting pigments: (i) two bis(phenylethynyl)anthracene chromophores, BPEA $a$ and $\mathrm{BPEA}_{b}$, (ii) two borondipyrromethene chromophores, $\mathrm{BDPY}_{a}$ and $\mathrm{BDPY}_{b}$, and (iii) two zinc tetraarylporphyrin chromophores, $\mathrm{ZnPy}_{a}$ and $\mathrm{ZnPy}_{b}$. All the chromophores are attached to a rigid hexaphenyl benzene core. In addition to the antenna components, the photosystem contains a fullerene derivative $(\mathrm{F})$ containing two pyridyl groups, acting as an electron acceptor. The fullerene derivative $\mathrm{F}$ is attached to the both $\mathrm{ZnPy}$ chromophores via the coordination of the pyridyl nitrogens with the zinc atoms. For structural details of the BPF complex, we refer to Refs. 17 and 28.

be considered as individual interacting units, but not as an extended single chromophore. We can expect that, at these conditions, quantum coherence is able to play an important role in energy and charge-transfer dynamics, manifesting itself in quantum beatings of chromophore populations as well as in quantum oscillations of the charge accumulated on the fullerene molecule. In principle, these oscillations could be measured by a sensitive single-electron transistor, thus providing a direct proof of quantum behavior in the artificial photosynthetic complex. Since these phenomena occurs at very short time scales (a few femtoseconds), they could be within the reach of femtosecond spectroscopy in the near future. The main goal of this study is to explore quantum features of the energy and charge transfer in a wheel-shaped antennareaction center complex at subpicosecond time scales.

\section{MODEL AND METHODS}

\section{A. Hamiltonian}

Each chromophore has one ground and one excited state, whereas the electron-acceptor fullerene $F$ has just one energy level with energy $E_{F}$. We introduce creation (annihilation) operators, $a_{k}^{\dagger}\left(a_{k}\right)$, of an electron on the $k$ th site. The electron population operators are defined as $n_{k}=a_{k}^{\dagger} a_{k}$. We assume that each electron state can be occupied by a single electron, as spin degrees of freedom are neglected. The basic Hamiltonian of the system has the form

$$
\begin{aligned}
H_{0}= & \sum_{k}\left(E_{k} n_{k}+E_{k^{*}} n_{k^{*}}\right)+E_{F} n_{F}+H_{C} \\
& +\sum_{k \neq l} V_{k l} a_{k^{*}}^{\dagger} a_{k} a_{l}^{\dagger} a_{l^{*}}-\sum_{\sigma \sigma^{\prime}} \Delta_{\sigma \sigma^{\prime}} a_{\sigma}^{\dagger} a_{\sigma^{\prime}},
\end{aligned}
$$

where the first part incorporates the energies of the electron states (hereafter $k, l=\mathrm{BPEA}_{a}, \mathrm{BPEA}_{b}, \mathrm{BDPY}_{a}, \mathrm{BDPY}_{b}$, $\mathrm{ZnPy}_{a}, \mathrm{ZnPy}_{b}$ ), and the second term is related to a fullerene energy level $E_{F}$ with a population operator $n_{F}=a_{F}^{\dagger} a_{F}$. The pair $\left(k, k^{*}\right)$ denotes a ground $(k)$ and an excited $\left(k^{*}\right)$ state of an electron located on the site $k$ with the corresponding energy $E_{k}\left(E_{k^{*}}\right)$. The term $H_{C}$ represents the contribution of Coulomb interactions between electron-binding sites. This term is given in Appendix A. The fourth term of Eq. (1) describes excitonic couplings between the chromophores $k$ and $l$. The matrix element $V_{k l}$ is a measure of an interchromophoric coupling strength. The last term in Eq. (1) describes the electron tunneling from excited states of the porphyrin molecules $\left(\mathrm{ZnPy}_{a}\right.$ and $\left.\mathrm{ZnPy}_{b}\right)$ to the electronacceptor $\mathrm{F}$ characterized by the tunneling amplitudes $\Delta_{\sigma \sigma^{\prime}}$, where $\sigma, \sigma^{\prime}=\mathrm{ZnPy}_{a}^{*}, \mathrm{ZnPy}_{b}^{*}, \mathrm{~F}$.

The interaction of the system with the environment (heat bath), represented here by a sum of independent oscillators with Hamiltonian

$$
H_{\mathrm{env}}=\sum_{j}\left(\frac{p_{j}^{2}}{2 m_{j}}+\frac{m_{j} \omega_{j}^{2} x_{j}^{2}}{2}\right),
$$

is given by the term

$$
H_{e-\mathrm{ph}}=-\sum_{j k} m_{j} \omega_{j}^{2} x_{j k} x_{j} n_{k},
$$

where $x_{j}$ and $p_{j}$ are the position and momentum of the $j$ th oscillator having an effective mass $m_{j}$ and a frequency $\omega_{j}$. The coefficients $x_{j k}$ define the strength of the coupling between the electron subsystem and the environment.

The contribution of the energy-quenching mechanisms responsible for the recombination processes in the system is given by the Hamiltonian,

$$
H_{\text {quen }}=-\sum_{l}\left(q_{l}^{\dagger} a_{l^{*}}^{\dagger} a_{l}+q_{l} a_{l}^{\dagger} a_{l^{*}}\right) .
$$

For the sake of simplicity, we include the radiation damping of the excited states into the energy-quenching operator $q_{l}$. The first term in the Hermitian Hamiltonian $H_{\text {quen }}$ is related to the excitation of the $l$-chromophore by the quenching bath, whereas the second term corresponds to the reverse process, namely, to the absorption of chromophore energy by the bath. Both processes are necessary to provide correct conditions for the thermodynamic equilibrium between the system and the bath.

The total Hamiltonian of the system is

$$
H=H_{0}+H_{e-\mathrm{ph}}+H_{\mathrm{env}}+H_{\text {quen }} .
$$

We omit here the Hamiltonian of the quenching (radiation) heat bath.

\section{B. Diagonalization of $\boldsymbol{H}_{0}$}

We choose 160 basis states $|M\rangle$ of the complex including a vacuum state, where all chromophores are in the ground state and the F site is empty. We diagonalize the Hamiltonian $H_{0}$ in Eq. (1) to consider the case where the excitonic coupling between chromophores, described by coefficients $V_{l m}$, 
and the porphyrin-fullerene tunneling, which is determined by amplitudes $\Delta_{\sigma \sigma^{\prime}}$, cannot be analyzed within perturbation theory. In the new basis, $|\mu\rangle=\sum_{M}|M\rangle\langle M \mid \mu\rangle$, the Hamiltonian $H_{0}$ is diagonal with the energy spectrum $\left\{E_{\mu}\right\}$, so that the total Hamiltonian of the system $H$ has the form

$$
H=\sum_{\mu} E_{\mu}|\mu\rangle\left\langle\mu\left|-\sum_{\mu \nu} \mathcal{A}_{\mu \nu}\right| \mu\right\rangle\langle\nu|+H_{\mathrm{env}} .
$$

Here

$$
\mathcal{A}_{\mu \nu}=Q_{\mu \nu}+q_{\mu \nu}
$$

is the combined operator for both heat baths with fluctuating in time variables:

$$
\begin{aligned}
Q_{\mu \nu}= & \sum_{j} m_{j} \omega_{j}^{2} x_{j}\left[x_{j F}\left\langle\mu\left|n_{F}\right| \nu\right\rangle\right. \\
& \left.+\sum_{k}\left(x_{j k}\left\langle\mu\left|n_{k}\right| \nu\right\rangle+x_{j k^{*}}\left\langle\mu\left|n_{k^{*}}\right| v\right\rangle\right)\right], \\
& q_{\mu \nu}=\sum_{l}\left\langle\mu\left|a_{l}^{\dagger} a_{l^{*}}\right| v\right\rangle q_{l}+\text { H.c. }
\end{aligned}
$$

To distinguish the processes of energy transfer, where the number of electrons on each chromophore remains constant, from the processes of charge transfer, where the total population of the site changes, we introduce the following operators:

$$
S_{l}=n_{l}+n_{l^{*}}, \quad M_{l}=n_{l}-n_{l^{*}},
$$

together with coefficients

$$
\bar{x}_{j l}=\frac{x_{j l}+x_{j l^{*}}}{2}, \quad \tilde{x}_{j l}=\frac{x_{j l}-x_{j l^{*}}}{2} .
$$

Thus, the environment operator $Q_{\mu \nu}$ can be rewritten as

$$
Q_{\mu \nu}=\sum_{j} m_{j} \omega_{j}^{2} x_{j} \Lambda_{j}^{\mu \nu}
$$

with

$$
\Lambda_{j}^{\mu \nu}=\sum_{l}\left\{\bar{x}_{j l}\left\langle\mu\left|S_{l}\right| v\right\rangle+\tilde{x}_{j l}\left\langle\mu\left|M_{l}\right| v\right\rangle\right\}+x_{j F}\left\langle\mu\left|n_{F}\right| v\right\rangle .
$$

\section{Non-Markovian equations for the system operators}

An arbitrary electron operator $W$ can be expressed in terms of the basic operators $\rho_{\mu \nu}=|\mu\rangle\langle\nu|$; with $W$ $=\sum_{\mu \nu} W_{\mu \nu} \rho_{\mu \nu}$, and $W_{\mu \nu}=\langle\mu|W| \nu\rangle$. The operator $\rho_{\mu \nu}$ denotes a matrix with zero elements, with the exception of the single element at the crossing of the $\mu$-row and the $\nu$-column. The matrix elements $W_{\mu \nu}$ of any electron operator can be easily calculated (see, e.g., Eqs. (S10) and (S11) in the supplementary material for Ref. 20). For example, an electron localized in a two-well potential, ${ }^{21}$ with the right and left states $|1\rangle$ and $|2\rangle$, is described by the Pauli matrices $\left\{\sigma_{x}, \sigma_{y}, \sigma_{z}\right\}: \sigma_{z}=|1\rangle\langle 1|-| 2\rangle\langle 2|, \sigma_{x}=$ $|1\rangle\langle 2|+| 2\rangle\langle 1|$, and $\sigma_{y}=i(|2\rangle\langle 1|-| 1\rangle\langle 2|)$, which are expressed in terms of the basic operators $|\mu\rangle\langle\nu|$ with $\mu, v$ $=1,2$.
In the Heisenberg picture, the operator $W$ evolves in time according to the equation: $i(\partial W / \partial t)=[W, H]_{-}$. This evolution can be described with the time-evolving operators, $\rho_{\mu \nu}(t)=(|\mu\rangle\langle\nu|)(t)$, which satisfy the Heisenberg equation:

$$
i \frac{\partial \rho_{\mu \nu}}{\partial t}=\left[\rho_{\mu \nu}, H\right]_{-}=-\omega_{\mu \nu} \rho_{\mu \nu}-\sum_{\alpha}\left(\mathcal{A}_{\nu \alpha} \rho_{\mu \alpha}-\mathcal{A}_{\alpha \mu} \rho_{\alpha \nu}\right),
$$

where $\omega_{\mu \nu}=E_{\mu}-E_{v}$, and the heat-bath operator $\mathcal{A}_{\mu \nu}$ is defined in Eq. (7). Here, we use the fact that the Hamiltonian $H$ in Eq. (6) is also expressed in terms of the operators $\rho_{\mu \nu}$ taken at the same moment of time $t$. For two of these operators, $\rho_{\mu \nu}(t)$ and $\rho_{\alpha \beta}(t)$, we have simple multiplication rules: $\rho_{\mu \nu} \rho_{\alpha \beta}=\delta_{\nu \alpha} \rho_{\mu \beta}$. These rules allow to calculate commutators of basic operators taken at the same moment of time. We note that at the initial moment of time the operator, $\rho_{\mu \nu}(0) \equiv|\mu\rangle\langle\nu|$, is represented by the above-mentioned zero matrix with a single unit at the $\mu-v$ intersection. The matrix elements of the electron operators in Eqs. (9) and (13) are taken over the time-independent eigenstates of the Hamiltonian $H_{0}$. The bath operators $\mathcal{A}_{\mu \nu}$ fluctuate in time since they depend on the environmental variables, $\left\{x_{j}(t)\right\}$, and on the variables $\left\{q_{l}(t)\right\}$ of the quenching bath.

It is known that the dissipative evolution of the twostate system can be described by the Heisenberg equations for the Pauli matrices $\left\{\sigma_{x}, \sigma_{y}, \sigma_{z}\right\}$ with the spin-boson Hamiltonian [see Eq. (1.4) in Ref. 21], which includes environmental degrees of freedom. The artificial photosynthetic complex analyzed in the present paper has 160 states. A dissipative evolution of this complex is described by the Hamiltonian $H$ in Eq. (6), written in terms of the Heisenberg operators $\rho_{\mu \nu}(t)=(|\mu\rangle\langle\nu|)(t)$ taken at the moment of time $t$. Instead of the time-dependent Pauli matrices, the time evolution of the two-state dissipative system can be described by the basic operators $|1\rangle\langle 1|| 1\rangle,\langle 2|| 2\rangle,\langle 1|| 2\rangle,\langle 2|$, evolving in time. In a similar manner, ${ }^{22}$ the evolution of the multi-state photosynthetic complex is described by the set of the time-dependent Heisenberg operators $\rho_{\mu v}(t)$, which obey Eq. (14). As its spinboson counterpart, the Hamiltonian $H$ in Eq. (6) contains the Hamiltonian, $H_{\text {env }}$, of the heat-bath as well as the system-bath interaction terms. Here, we generalize the spin-boson model from the case of two states to the case of 160 states. With a knowledge of the operators $\rho_{\mu \nu}(t)$, it is possible to find the time evolution of any Heisenberg operator of the system. Only at the initial moment of time, $t=0$, the operators $\rho_{\mu \nu}(0)$ form the basis of the Liouville space. Note that we work in the Heisenberg representation, without using the description based on the von Neumann equations for the density matrix.

To obtain functions that can be measured in experiments, we have to average the operator $\rho_{\mu \nu}(t)$ and Eq. (14) over the initial state $\left|\Psi^{0}\right\rangle$ of the electron subsystem as well as over the Gaussian distribution, $\rho_{T}=\exp \left(-H_{\text {bath }}^{(0)} / T\right)$, of the equilibrium bath, $\langle\ldots\rangle_{T}$, with temperature $T$ and with a free Hamiltonian $H_{\text {bath }}^{(0)}$, which comprises the free environment Hamiltonian and the free Hamiltonian of the quenching bath. The notation $\langle\ldots\rangle$ means double averaging:

$$
\langle\ldots\rangle=\left\langle\left\langle\Psi^{0}|\ldots| \Psi^{0}\right\rangle\right\rangle_{T} .
$$


The quantum-mechanical average value of the initial basic matrix, $\left\langle\Psi^{0}\left|\rho_{\mu \nu}(0)\right| \Psi^{0}\right\rangle=\left\langle\Psi^{0} \mid \mu\right\rangle\left\langle\nu \mid \Psi^{0}\right\rangle$, is determined by the product of amplitudes to find the electron subsystem at the initial moment of time in the eigenstates $|\mu\rangle$ and $|\nu\rangle$ of the Hamiltonian $H_{0}$.

A standard density matrix, $\bar{\rho}=\left\{\bar{\rho}_{\mu \nu}\right\}$, of the electron subsystem is a deterministic function which allows to calculate the average value of an arbitrary operator $W$ with the formula:

$$
\langle W(t)\rangle=\operatorname{Tr}[\bar{\rho}(t) W]=\sum_{\mu \nu} W_{\mu \nu} \bar{\rho}_{\nu \mu}(t) .
$$

The same average value can be written as $\langle W(t)\rangle=$ $\sum_{\mu \nu} W_{\mu \nu}\left\langle\rho_{\mu \nu}(t)\right\rangle$, which means that the average matrix, $\left\langle\rho_{\mu \nu}(t)\right\rangle=\bar{\rho}_{\nu \mu}(t)$, has matrix elements related to the transposed density matrix $\bar{\rho}(t)$.

It should be emphasized that the time evolution of the heat-bath operators $\left\{x_{j}, p_{j}\right\}$ and $\left\{q_{l}\right\}$, as well as their linear combinations $Q_{\mu \nu}, q_{\mu \nu}$, and $\mathcal{A}_{\mu \nu}$, are determined by the total Hamiltonian $H$ in Eq. (6). In the absence of an interaction with the dynamical system (the electron-binding sites), the free-phonon operators $Q_{\mu \nu}^{(0)}$, as well as the free operators of the other baths, $q_{\mu \nu}^{(0)}$, are described by Gaussian statistics, ${ }^{23}$ as in the case of an environment comprising independent linear oscillators with the Hamiltonian $H_{\text {env }}$ in Eq. (2). Using Gaussian property, Efremov et al. ${ }^{24}$ derived non-Markovian Heisenberg-Langevin equations, without using perturbation theory, that assumes a weak system-bath interaction. Recently, a similar non-perturbative approach has been developed by Ishizaki and Fleming in Ref. 25. Due to Gaussian properties of the free bath, the total operator $\mathcal{A}_{\mu \nu}$ of the combined dissipative environment is a linear functional of the operators $\rho_{\mu \nu}$,

$$
\begin{aligned}
\mathcal{A}_{\mu \nu}(t)= & \mathcal{A}_{\mu \nu}^{(0)}(t) \\
& +\sum_{\bar{\mu} \bar{\nu}} \int d t_{1}\left\langle i\left[\mathcal{A}_{\mu \nu}^{(0)}(t), \mathcal{A}_{\bar{\mu} \bar{\nu}}^{(0)}\left(t_{1}\right)\right]_{-}\right\rangle \theta\left(t-t_{1}\right) \rho_{\bar{\mu} \bar{\nu}}\left(t_{1}\right),
\end{aligned}
$$

where $\theta(\tau)$ is the Heaviside step function. We note that this expansion directly follows from the solution of the Heisenberg equations for the positions $\left\{x_{j}\right\}$ and $\left\{q_{l}\right\}$ of the bath oscillators. It is shown in Ref. 24 that the average value of the free operator $\mathcal{A}_{\mu \nu}^{(0)}(t)$ multiplied by an arbitrary operator $\mathcal{B}(t)$ is proportional to the functional derivative of the operator $\mathcal{B}$ over the variable $\mathcal{A}_{\mu \nu}^{(0)}(t)$ :

$$
\left\langle\mathcal{A}_{\mu \nu}^{(0)}(t) \mathcal{B}(t)\right\rangle=\sum_{\bar{\mu} \bar{\nu}} \int d t_{1}\left\langle\mathcal{A}_{\mu \nu}^{(0)}(t) \mathcal{A}_{\bar{\mu} \bar{\nu}}^{(0)}\left(t_{1}\right)\right\rangle \times\left\langle\frac{\delta \mathcal{B}(t)}{\delta \mathcal{A}_{\bar{\mu} \bar{\nu}}^{(0)}\left(t_{1}\right)}\right\rangle,
$$

with

$$
\frac{\delta \mathcal{B}(t)}{\delta \mathcal{A}_{\bar{\mu} \bar{\nu}}^{(0)}\left(t_{1}\right)}=i\left[\mathcal{B}(t), \rho_{\bar{\mu} \bar{\nu}}\left(t_{1}\right)\right]_{-} \theta\left(t-t_{1}\right) .
$$

Substituting Eqs. (17)-(19) into Eq. (14) we derive the exact non-Markovian equation for the Heisenberg operators $\rho_{\mu \nu}$ of the dynamical system (chromomorphic sites + fullerene) interacting with a Gaussian heat bath,

$$
\begin{aligned}
\left\langle\dot{\rho}_{\mu \nu}\right\rangle-i \omega_{\mu \nu}\left\langle\rho_{\mu \nu}\right\rangle= & \sum_{\alpha \bar{\mu} \bar{\nu}} \int_{0}^{t} d t_{1}\left\{\left\langle\mathcal{A}_{\bar{\mu} \bar{\nu}}^{(0)}\left(t_{1}\right) \mathcal{A}_{\nu \alpha}^{(0)}(t)\right\rangle\left\langle\rho_{\bar{\mu} \bar{\nu}}\left(t_{1}\right) \rho_{\mu \alpha}(t)\right\rangle\right. \\
& -\left\langle\mathcal{A}_{\nu \alpha}^{(0)}(t) \mathcal{A}_{\bar{\mu} \bar{\nu}}^{(0)}\left(t_{1}\right)\right\rangle\left\langle\rho_{\mu \alpha}(t) \rho_{\bar{\mu} \bar{\nu}}\left(t_{1}\right)\right\rangle \\
& +\left\langle\mathcal{A}_{\alpha \mu}^{(0)}(t) \mathcal{A}_{\bar{\mu} \bar{\nu}}^{(0)}\left(t_{1}\right)\right\rangle\left\langle\rho_{\alpha \nu}(t) \rho_{\bar{\mu} \bar{\nu}}\left(t_{1}\right)\right\rangle \\
& \left.-\left\langle\mathcal{A}_{\bar{\mu} \bar{\nu}}^{(0)}\left(t_{1}\right) \mathcal{A}_{\alpha \mu}^{(0)}(t)\right\rangle\left\langle\rho_{\bar{\mu} \bar{\nu}}\left(t_{1}\right) \rho_{\alpha \nu}(t)\right\rangle\right\} .
\end{aligned}
$$

The time evolution of the average operator $\left\langle\rho_{\mu \nu}\right\rangle$ is determined by the second-order correlation functions of the system operators as well as by the correlation functions of the free dissipative environment. Here, we do not impose any restrictions on the spectrum of the environment. It should be emphasized that the exact non-Markovian equation (20) goes far beyond the von Neumann equation, $i \dot{\bar{\rho}}=[H, \bar{\rho}]_{-}$, for the density matrix $\bar{\rho}$ of the electron subsystem.

\section{Beyond the system-bath perturbation theory}

We assume that the coupling of the system to the quenching heat bath determined by the Hamiltonian $H_{\text {quen }}$ in Eq. (4) is weak enough to be analyzed perturbatively. However, an interaction of the chromophores with the protein environment cannot be treated entirely within perturbation theory since the reorganization energies are of the order of the intersite couplings. As in the theory of modified Redfield equations, ${ }^{26,27}$ the phonon operator $Q_{\mu \nu}$ in Eq. (12) can be represented as a sum of diagonal $Q_{\mu}=Q_{\mu \mu}$ and off-diagonal $\tilde{Q}_{\mu \nu}$ parts:

$$
Q_{\mu \nu}=Q_{\mu} \delta_{\mu \nu}+\left(1-\delta_{\mu \nu}\right) \tilde{Q}_{\mu \nu} .
$$

We derive equations for diagonal and off-diagonal elements of the matrix $\left\langle\rho_{\mu \nu}(t)\right\rangle$ (see Appendix B for details about the derivation), where the interaction with the off-diagonal elements of the environment operators $\tilde{Q}_{\mu \nu}$ are considered within the perturbation theory, and the effects of the diagonal elements $Q_{\mu}$ are treated exactly.

The time dependence of the electron distribution $\left\langle\rho_{\mu}\right\rangle$ (diagonal elements) over eigenstates of the Hamiltonian $H_{0}$ is governed by the equation

$$
\left\langle\dot{\rho}_{\mu}\right\rangle+\gamma_{\mu}\left\langle\rho_{\mu}\right\rangle=\sum_{\alpha} \gamma_{\mu \alpha}\left\langle\rho_{\alpha}\right\rangle
$$

where the relaxation matrix $\gamma_{\mu \alpha}$ contains a contribution, $\tilde{\gamma}_{\mu \alpha}$, from the non-diagonal environment operators [see Eq. (B22)] as well as a contribution from the quenching processes, $\gamma_{\mu \alpha}^{\text {quen }}$ [see Eq. (B30)],

$$
\gamma_{\mu \alpha}=\tilde{\gamma}_{\mu \alpha}+\gamma_{\mu \alpha}^{\text {quen }}
$$

with the total relaxation rate $\gamma_{\mu}=\sum_{\alpha} \gamma_{\alpha \mu}$. The time evolution of the off-diagonal elements are given by Eq. (B31) in Appendix B.

Equations (22) and (B31) allow us to determine the time evolution of an average value for an arbitrary operator $W$ of the system: $\langle W(t)\rangle=\sum_{\mu \nu}\langle\mu|W| v\rangle\left\langle\rho_{\mu \nu}(t)\right\rangle$. 


\section{ENERGIES AND OTHER PARAMETERS}

\section{A. Energy levels and electrochemical potentials}

The energies of the excited states of chromophores BPEA, BDPY, and $\mathrm{ZnPy}$, in the BPF complex are estimated from the average between the longest wavelength absorption band and the shortest wavelength emission band of the chromophores. The average excited state energies of the chromophores BPEA, BDPY, and ZnPy are $2610 \mathrm{meV}, 2370$ $\mathrm{meV}$, and $2030 \mathrm{meV}$, respectively, if we count from the corresponding ground energy levels. ${ }^{16,17}$ Cyclic voltammetric studies ${ }^{17}$ of reduction potentials with respect to the standard calomel electrode show that the first reduction potential of the fullerene derivative, $\mathrm{F}$, is about $-0.62 \mathrm{~V}$ and the first oxidation potential of $\mathrm{ZnPy}$ is about $0.75 \mathrm{~V}$. From these data we calculate that the energy of the charge-separated state $\mathrm{ZnPy}^{+}-\mathrm{F}^{-}$ is about $1370 \mathrm{meV}$. This energy is the sum of the energy of an electron on site $\mathrm{F}$ and the Coulomb interaction energy between a positive charge on $\mathrm{ZnPy}$ and a negative charge on F. The Coulomb energy can be calculated with the formula $u=e^{2} / 4 \pi \epsilon_{0} \epsilon r$, where $\epsilon_{0}$ is the vacuum dielectric constant. The dielectric constant $\epsilon$ of 1,2-diflurobenzene (a solvent used in all experimental measurements of Ref. 17) is about 13.8. If the distance $r$ between porphyrin $\mathrm{ZnPy}$ and fullerene $\mathrm{F}$ is about $1 \mathrm{~nm}$, the Coulomb interaction energy is about $105 \mathrm{meV}$. Thus, the estimated energy of the electron on F can be of the order of $1475 \mathrm{meV}$.

\section{B. Reorganization energies and coupling strengths}

The reorganization energies for exciton and electron transfer processes and electronic coupling strengths between the chromophores depend on the mutual distances and orientations of the components, strengths of chemical bonds, solvent polarity, and other structural details of the system. Precise values of these parameters are not available. However, time constants for energy transfer between different chromophores in the BPF complex, as well as rates for transitions of electrons between the fullerene $\mathrm{F}$ and porphyrin chromophores $\mathrm{ZnPy}$, have been reported in Ref. 17. We fit
TABLE II. Comparison between the calculated values of the time constants (using the parameter sets I and II) to the experimental values reported in Ref. 17.

\begin{tabular}{lllc}
\hline \hline Process & $\tau$ (Set I) & $\tau$ (Set II) & $\tau$ (Experimental) \\
\hline $\mathrm{BPEA}_{\mathrm{a}} \rightarrow \mathrm{BPEA}_{\mathrm{b}}$, & $\sim 0.4 \mathrm{ps}$ & $\sim 0.2 \mathrm{ps}$ & $0.4 \mathrm{ps}$ \\
$\mathrm{BPEA}_{b} \rightarrow \mathrm{BPEA}_{a}$ & & \\
$\mathrm{BPEA}_{\mathrm{a}} \rightarrow \mathrm{BDPY}_{\mathrm{a}}$, & $\sim 5 \mathrm{ps}$ & $\sim 5.4 \mathrm{ps}$ & $5-13 \mathrm{ps}$ \\
$\mathrm{BPEA}_{b} \rightarrow \mathrm{BDPY}_{b}$ & & \\
$\mathrm{BDPY}_{\mathrm{a}} \rightarrow \mathrm{ZnPy}_{\mathrm{a}}$, & $\sim 5 \mathrm{ps}$ & $\sim 3.9 \mathrm{ps}$ & $2-15 \mathrm{ps}$ \\
$\mathrm{BDPY}_{b} \rightarrow \mathrm{ZnPy}_{b}$ & & \\
$\mathrm{BPEA}_{\mathrm{a}} \rightarrow \mathrm{ZnPy}_{\mathrm{a}}$, & $\sim 12 \mathrm{ps}$ & $\sim 12 \mathrm{ps}$ & $7 \mathrm{ps}$ \\
$\mathrm{BPEA}_{b} \rightarrow \mathrm{ZnPy}_{b}$ & & \\
$\mathrm{BPEA}_{\mathrm{b}} \rightarrow \mathrm{ZnPy}_{\mathrm{a}}$, & $\sim 10 \mathrm{ps}$ & $\sim 12 \mathrm{ps}$ & $6 \mathrm{ps}$ \\
$\mathrm{BPEA}_{a} \rightarrow \mathrm{ZnPy}$ & & \\
$\mathrm{ZnPy}_{b} \rightarrow \mathrm{F}$, & & $\sim 3 \mathrm{ps}$ & $3 \mathrm{ps}$ \\
$\mathrm{ZnPy}_{a} \rightarrow \mathrm{F}$ & $\sim 3 \mathrm{ps}$ &
\end{tabular}

the experimental values of these time constants with the rates following from our equations with the goal of extracting reasonable values for the reorganization energies and the electronic and excitonic couplings. In principle, many combinations of reorganization energies and coupling constants could be possible. For the sake of simplicity, we consider two sets of parameters, for two limiting situations. One parameter set (denoted by set I in Table I) corresponds to a larger excitonic couplings, $V$, compared to the reorganization energies, $\Lambda$, whereas another set of parameters (denoted by set II in Table I) considers the opposite case, where the reorganization energies are larger than the excitonic couplings. These two sets of parameters are presented in Table I. In addition to the parameters listed in Table I, we consider the following values for the charge-transfer reorganization energies (set I): $\lambda_{F}=200 \mathrm{meV}, \lambda_{l M}=100 \mathrm{meV}$, and $\lambda_{F}=230 \mathrm{meV}$, $\lambda_{l M}=120 \mathrm{meV}$ (set II), where $l=\mathrm{ZnPy}_{a}, \mathrm{ZnPy}_{b}$. The values of the reorganization energies for energy-transfer processes are much smaller than those for charge transfer.

References 17 and 28 reported a very fast electron transfer (with a time constant $\tau \sim 3$ ps) between excited states of zinc porphyrins $\left(\mathrm{ZnPy}_{a}, \mathrm{ZnPy}_{b}\right)$ and the fullerene derivative $\mathrm{F}$. This fact indicates a good porphyrin-fullerene

TABLE I. Chosen values of the excitonic couplings $(V)$ and reorganization energies for energy transfer $(\Lambda)$ of the six antenna chromophores. We choose two sets of parameters, one set (denoted by I) corresponds to $V>\Lambda$ and the other set (II) to the opposite limit $V<\Lambda$. The calculated values of the time constants using both sets of parameters agree with the experimental values.

\begin{tabular}{|c|c|c|c|c|}
\hline \multirow[b]{2}{*}{ Chromophores } & \multicolumn{2}{|r|}{ Set I } & \multicolumn{2}{|r|}{ Set II } \\
\hline & Coupling $(V)$ & Reorganization energy $(\Lambda)$ & Coupling $(V)$ & Reorganization energy $(\Lambda)$ \\
\hline $\begin{array}{l}\mathrm{BPEA}_{a} \leftrightarrow \mathrm{BPEA}_{b} \\
\mathrm{BPEA}_{b} \leftrightarrow \mathrm{BPEA}_{a}\end{array}$ & $50 \mathrm{meV}$ & $\begin{aligned} \Lambda_{\mathrm{BPEA}_{a}} & =20 \mathrm{meV} \\
\Lambda_{\mathrm{BPEA}_{b}} & =20 \mathrm{meV}\end{aligned}$ & $30 \mathrm{meV}$ & $\begin{aligned} \Lambda_{\mathrm{BPEA}_{a}} & =40 \mathrm{meV} \\
\Lambda_{\mathrm{BPEA}_{b}} & =40 \mathrm{meV}\end{aligned}$ \\
\hline $\begin{array}{l}\mathrm{BPEA}_{a} \leftrightarrow \mathrm{BDPY}_{a} \\
\mathrm{BPEA}_{b} \leftrightarrow \mathrm{BDPY}_{b}\end{array}$ & $30 \mathrm{meV}$ & $\begin{array}{l}\Lambda_{\mathrm{BDPY}_{a}}=15 \mathrm{meV} \\
\Lambda_{\mathrm{BDPY}_{b}}=15 \mathrm{meV}\end{array}$ & $17 \mathrm{meV}$ & $\begin{array}{l}\Lambda_{\mathrm{BDPY}_{a}}=30 \mathrm{meV} \\
\Lambda_{\mathrm{BDPY}_{b}}=30 \mathrm{meV}\end{array}$ \\
\hline $\begin{array}{l}\mathrm{BDPY}_{a} \leftrightarrow \mathrm{ZnPy}_{a} \\
\mathrm{BDPY}_{b} \leftrightarrow \mathrm{ZnPy}_{b}\end{array}$ & $60 \mathrm{meV}$ & $\begin{aligned} \Lambda_{\mathrm{ZnPy}_{a}} & =20 \mathrm{meV} \\
\Lambda_{\mathrm{ZnPy}_{b}} & =20 \mathrm{meV}\end{aligned}$ & $25 \mathrm{meV}$ & $\begin{aligned} \Lambda_{\mathrm{ZnPy}_{a}} & =40 \mathrm{meV} \\
\Lambda_{\mathrm{ZnPy}_{b}} & =40 \mathrm{meV}\end{aligned}$ \\
\hline $\begin{aligned} \mathrm{BPEA}_{a} \leftrightarrow \mathrm{ZnPy}_{a} \\
\mathrm{BPEA}_{b} \leftrightarrow \mathrm{ZnPy}_{b}\end{aligned}$ & $50 \mathrm{meV}$ & $\ldots$ & $40 \mathrm{meV}$ & $\ldots$ \\
\hline $\begin{array}{l}\mathrm{BPEA}_{b} \leftrightarrow \mathrm{ZnPy}_{a} \\
\mathrm{BPEA}_{a} \leftrightarrow \mathrm{ZnPy}_{b}\end{array}$ & $60 \mathrm{meV}$ & $\cdots$ & $40 \mathrm{meV}$ & $\cdots$ \\
\hline
\end{tabular}


electronic coupling, which is due to the short covalent linkage and close spatial arrangement of the components. ${ }^{28}$ Hereafter, we assume that the $\mathrm{ZnPy}-\mathrm{F}$ tunneling amplitudes $\Delta$ are about $100 \mathrm{meV}$ (parameter set I) and $80 \mathrm{meV}$ (parameter set II). These parameters provide a quite fast electron transfer despite a significant energy gap between the $\mathrm{ZnPy}$ excited states and the fullerene energy level.

Using the parameter sets I and II we have calculated time constants for energy and charge processes. A comparison between the calculated and experimental values of the time constants has been presented in Table II.

To describe recombination processes, we introduce a coupling of the $l$ th chromophore to a quenching heat bath characterized for simplicity by the Ohmic spectral density: $\chi_{l}^{\prime \prime}(\omega)=\alpha_{l} \omega$ with a dimensionless constant $\alpha_{l}$. We assume that the shifts of the energy levels caused by the quenching bath are included into the renormalized parameters of the electron subsystem. The experimental values ${ }^{17,28}$ of the lifetimes $\tau_{l}^{e}$ for excited states of chromophores BPEA, BDPY, and ZnPy: $\tau_{\mathrm{BPEA}}^{e}=2.82 \mathrm{~ns}, \tau_{\mathrm{BDPY}}^{e}=0.26 \mathrm{~ns}$, and $\tau_{\mathrm{ZnPY}}^{e}=0.45 \mathrm{~ns}$, respectively, can be achieved with the following set of coupling constants: $\alpha_{\mathrm{BPEA}} \sim 10^{-7}, \alpha_{\mathrm{BDPY}}$ $\sim 10^{-6}$, and $\alpha_{\mathrm{ZnPy}} \sim 7 \times 10^{-7}$.

\section{RESULTS AND DISCUSSIONS}

Using Eqs. (B31) and (22) and two sets of parameters discussed in Sec. III, here we study electron and energy-transfer kinetics in the BPF complex with special emphasis on the femtosecond time range, where the effects of quantum coherence can play an important role. We consider both single- and double-exciton regimes.

\section{A. Evolution of a single exciton in the BPF complex}

In Fig. 2, we show the time evolution of the excited states populations provided that only the $\mathrm{BPEA}_{a}$ chromophore is excited at $t=0$ (single-exciton regime). We use here the parameter set I, where excitonic couplings are larger than reorganization energies (see Sec. III). The process starts with quantum beatings between the resonant $\mathrm{BPEA}_{a}$ and $\mathrm{BPEA}_{b}$ chromophores, with a decoherence time of the order of $100 \mathrm{fs}$ (at $T=300 \mathrm{~K}$ ). In a few picoseconds, the excitation energy is subsequently transferred to the adjacent BDPY moieties and to the $\mathrm{ZnPy}$ chromophores. Later on, an electron moves from the excited energy level of the porphyrins to the fullerene moiety, thus producing a charge-separated state, $\mathrm{ZnPy}^{+}-\mathrm{F}^{-}$, with a quantum yield $95 \%$, which is in agreement with the experimental results. ${ }^{16}$

It is evident from Fig. 2 that excited state populations of the BDPY chromophores oscillate with much lower amplitudes and die out within a very short time, $t<10 \mathrm{fs}$, at both temperatures: $T=300 \mathrm{~K}$ and $77 \mathrm{~K}$. The populations of the other sites of the BPF complex do not exhibit any oscillatory behavior. This can be ascribed to incoherent hopping becoming dominant because of significant energy mismatch between these chromophores.

Figure 3 shows the time dependence of the excited state populations of chromophores for the parameter set II, where the reorganization energies are larger than the excitonic cou-

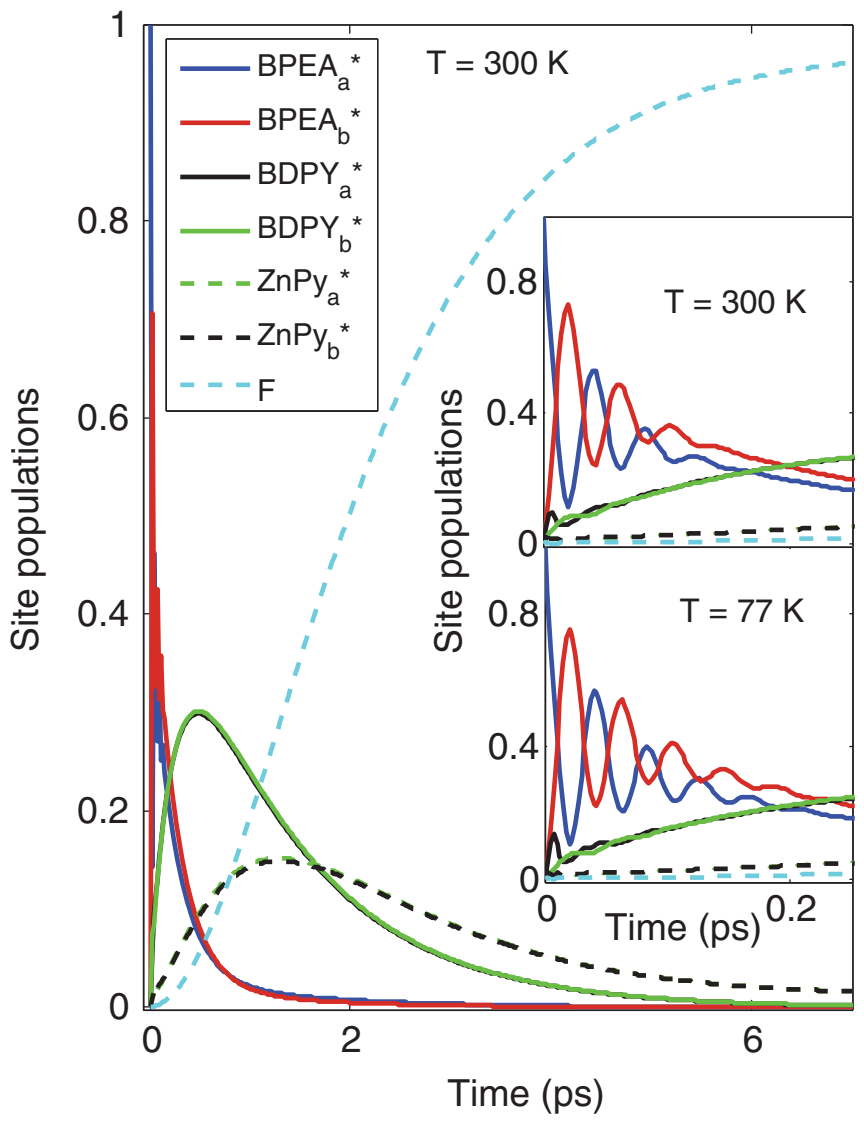

FIG. 2. Site populations as a function of time for the parameter set I. The inset plots depict the features of site populations for short time, at two different temperatures: $T=300 \mathrm{~K}$ and $77 \mathrm{~K}$. The site populations of the BPEA moieties oscillate with a considerably large amplitude, while the oscillations of the other site populations are hardly observable.

plings between chromophores. At $t=0$, the $\mathrm{BPEA}_{a}$ chromophore is excited (single-exciton regime). Then, after a few picoseconds, the charge-separated state is formed with a quantum yield of the order of $97 \%$. However, owing to a stronger system-environment coupling, quantum beats between the $\mathrm{BPEA}_{a}$ and $\mathrm{BPEA}_{b}$ chromophores have a lower amplitude and shorter decoherence time ( $\sim 50 \mathrm{fs})$ than in the previous case when we used the parameter set I. We note that no quantum oscillations of the fullerene population (site F) are visible in Figs. 2 and 3.

No significant oscillations of the site populations were observed (not shown here) when the BDPY chromophores were initially (at $t=0$ ) excited. In this case, due to the considerable energy gaps between the BDPY and the adjacent BPEA and $\mathrm{ZnPy}$ chromophores, incoherent hopping dominates over the coherent transfer of excitons. Furthermore, the structure of the BPF complex ${ }^{15,28}$ does not allow direct energy transfer between two BDPY chromophores.

Figures 4 and 5 demonstrate the charge- and energytransfer dynamics for two parameter sets, I and II, for the case when one of the porphyrin chromophores $\left(\mathrm{ZnPy}_{a}\right)$ is excited. Here, we do not show the time evolution of the BPEA and BDPY chromophores since these moieties have higher excitation energies than the $\mathrm{ZnPy}$ chromophore and they are not excited in the process. As evident from Figs. 4 and 5, the 


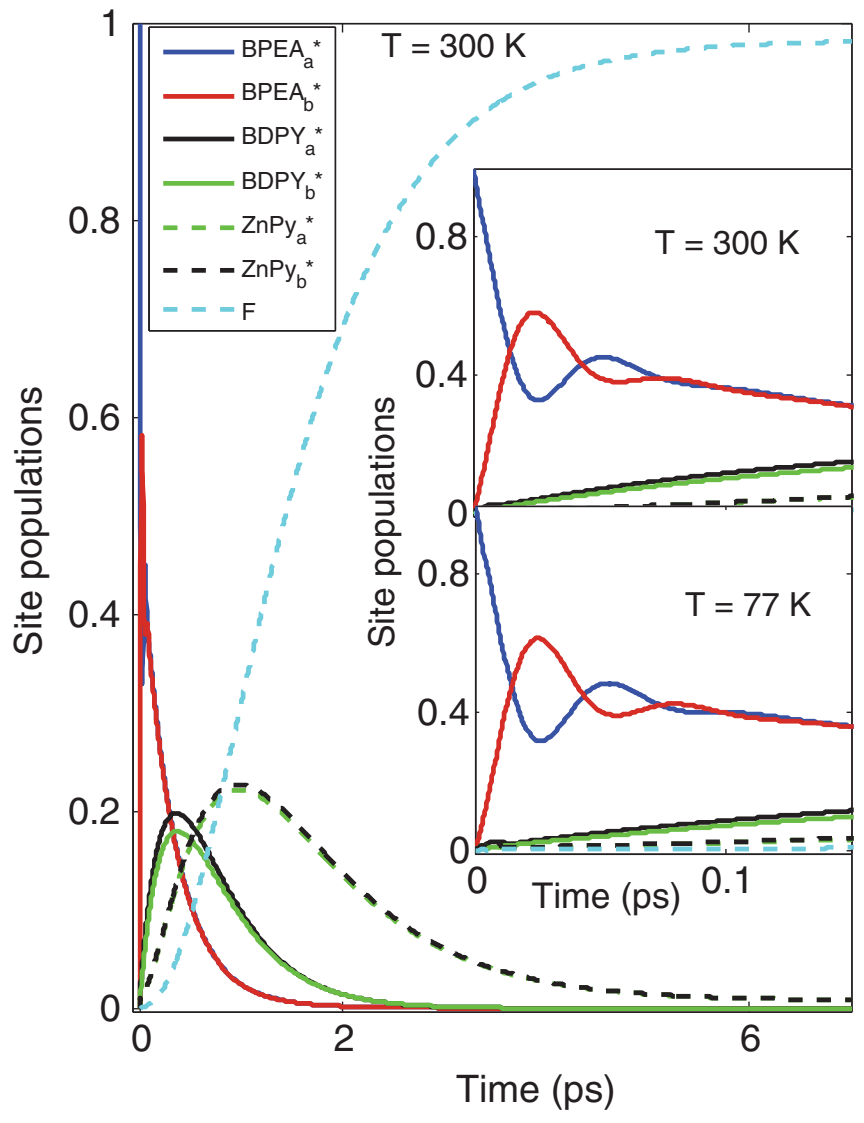

FIG. 3. This figure presents site populations as a function of time for the parameter set II. The inset plots show the site populations for short time, at two different temperatures: $T=300 \mathrm{~K}$ and $77 \mathrm{~K}$. The amplitudes of the site-population oscillations are much smaller and die out earlier, compared to Fig. 2. This figure indicates that even for $\Lambda>V$, the energy transfer between BPEA chromophores is dominated by wave-like coherent motion.

excited porphyrin molecule rapidly transfers an electron to fullerene, thus producing a charge-separated state $\mathrm{ZnPy}^{+}-\mathrm{F}^{-}$ with a quantum yield of about $98 \%$. The most important feature here is that the population and charge of the fullerene molecule oscillates in time due to a quantum superposition of the porphyrin excited state and the state of an electron on the fullerene. The amplitude of these quantum beats is very small and the decoherence time is quite short $(\sim 10 \mathrm{fs}$ at $\mathrm{T}=77$ $\mathrm{K})$. This fact can be explained by the significant energy mismatch between the $\mathrm{ZnPy}^{*}-\mathrm{F}$ and $\mathrm{ZnPy}^{+}-\mathrm{F}^{-}$states as well as by the strong influence of the environment on the electron dynamics.

\section{B. Evolution of double excitons in the BPF complex}

In Sec. IV A, we consider a single exciton case with just one chromophore initially being in the upper energy state. Here we analyze a situation where two porphyrin molecules $\left(\mathrm{ZnPy}_{a}\right.$ and $\left.\mathrm{ZnPy}_{b}\right)$ are excited at $t=0$. Figures 6(a) and 6(b) show the coherent dynamics of the fullerene population (and the fullerene charge) for the parameter sets I (Fig. 6(a)) and II (Fig. 6(b)) at two different temperatures, $T=77 \mathrm{~K}$ and $T=300 \mathrm{~K}$. We also compare the double-exciton case with the previously analyzed single-exciton case. It is apparent

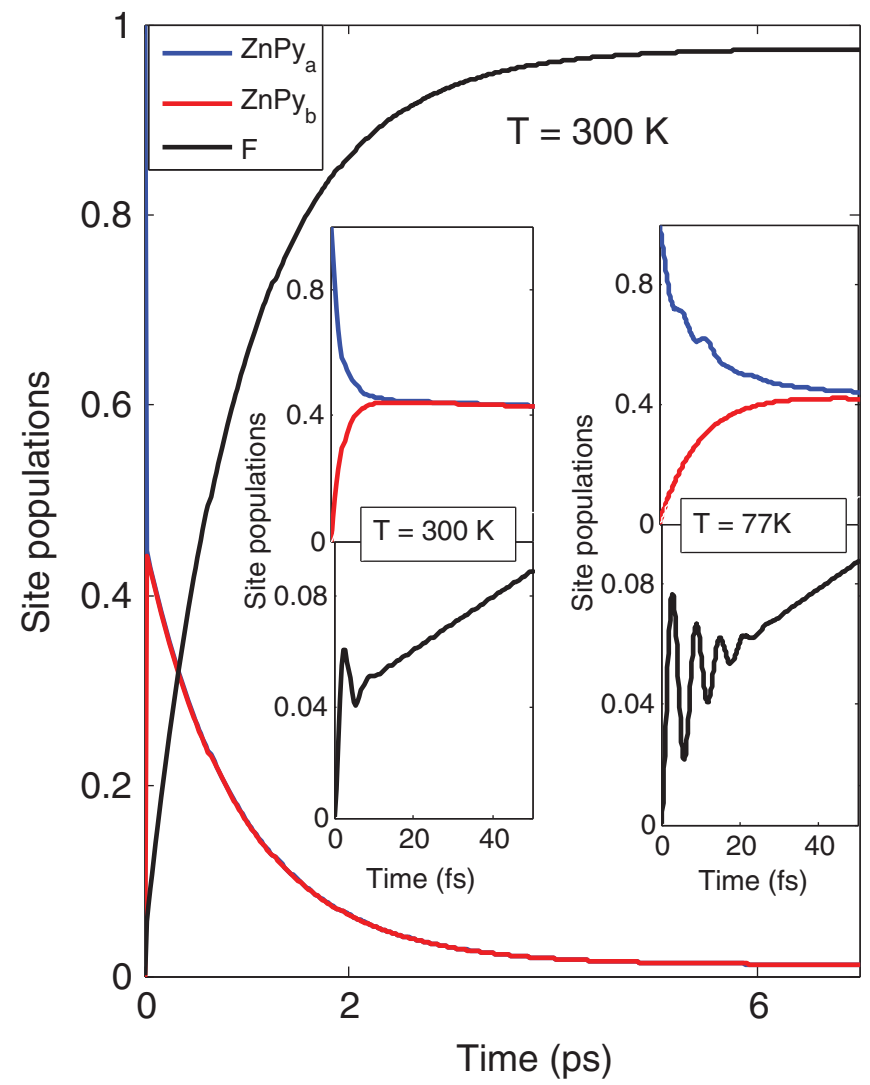

FIG. 4. Site populations as a function of time for the parameter set I, when the $\mathrm{ZnPy}_{a}$ chromophore is in the excited state and all the other chromophores are in the ground state at $t=0$. The inset plots depict the site populations at short time for two temperatures: $T=300 \mathrm{~K}$ and $77 \mathrm{~K}$. Lowering the temperature enhances the oscillations of the charge density on the fullerene moiety. Despite the huge energy difference between $\mathrm{ZnPy}^{*}-\mathrm{F}$ and $\mathrm{ZnPy}^{+}-\mathrm{F}^{-}$, the charge of the fullerene site exhibits oscillatory behavior for short time, specially at lower temperatures.

from Fig. 6 that the double excitation significantly enhances the amplitude of quantum oscillations of the fullerene charge for both sets of parameters. As one might expect, the frequency of the quantum beatings and the decoherence time are not affected by the number of excitons.

\section{Amplification of charge oscillations}

In the previous discussion we observed that lowering the temperature and the simultaneous excitation of both porphyrins significantly enhances quantum oscillations of the fullerene charge. In this subsection we show that these oscillations can also be controlled by tuning the following parameters:

\section{Electron tunneling amplitude $\Delta$}

The electronic coupling between the fullerene electron acceptor and zinc porphyrins has a strong effect on the quantum oscillations of the fullerene charge. To explore this effect, in Fig. 7(a) we plot the electron population of the fullerene as a function of time, for different values of the coupling $\Delta$. Figure 7(a) clearly shows that, with increasing $\Delta$, the ampli- 


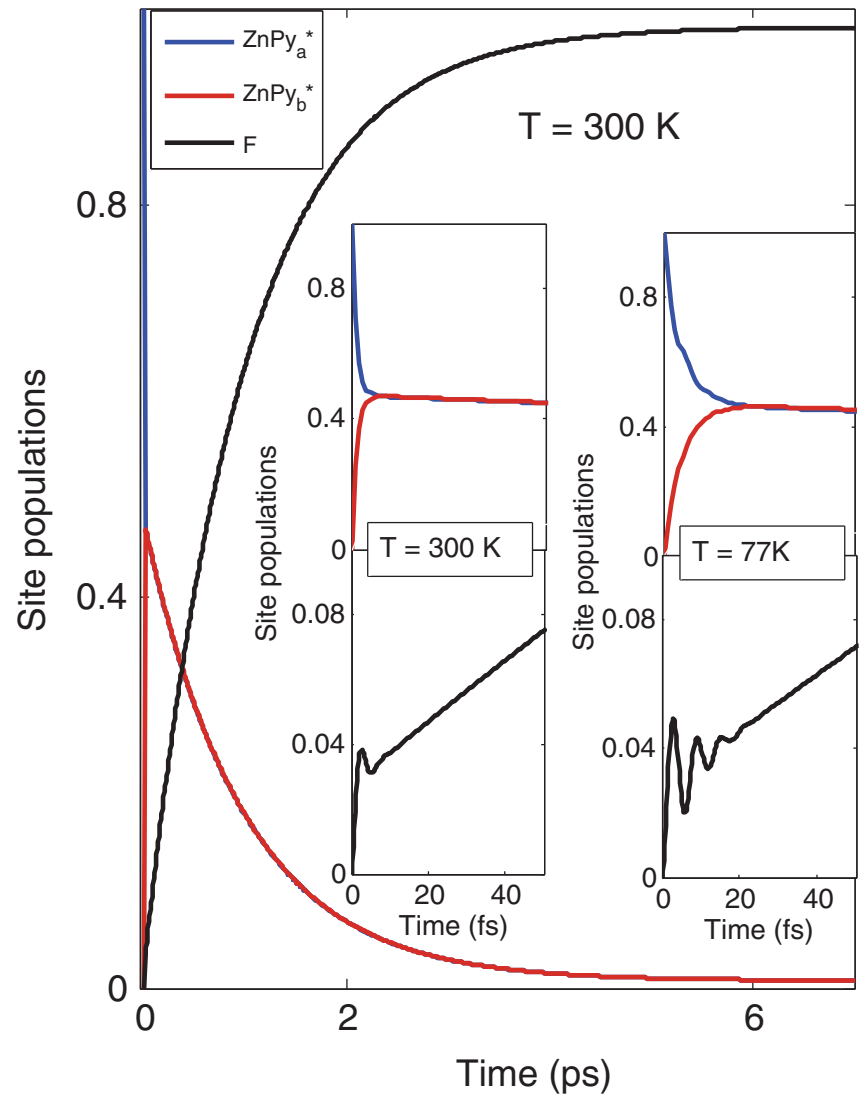

FIG. 5. Time evolution of the site populations for the parameter set II, starting with an exciton on the chromophore $\mathrm{ZnPy}_{a}$ at $t=0$. The inset plots depict the features of the site populations for a shorter time regime and at two temperatures: $T=300 \mathrm{~K}$ and $77 \mathrm{~K}$. Lowering the temperature enhances oscillations of the charge density on the fullerene derivative. These results indicate that the population of the site $\mathrm{F}$ oscillates for short time, even for $\Lambda>V$. These oscillations are more pronounced at lower temperatures.

tude of the charge oscillations is significantly enhanced. This coupling can be increased by attaching the fullerene to porphyrins with better ligands which form much stronger covalent bonds.

\section{Energy of the charge-separated state $E_{\mathrm{ch}}$}

The energy, $E_{\mathrm{ch}} \sim 1370 \mathrm{meV}$, of the charge-separated state, $\mathrm{ZnPy}^{+}-\mathrm{F}^{-}$, is much lower than the energy of the zinc porphyrin excited state, $E_{\mathrm{ZnPy}^{*}} \sim 2030 \mathrm{meV}$. It is evident from Fig. 7(b) that increasing the energy $E_{\mathrm{ch}}$, which leads to a decrease of the porpyrin-fullerene energy mismatch, results in a pronounced amplification of the quantum oscillations of the fullerene charge. The energy of the fullerene can be changed by placing nearby a charge residue, electrostatically coupled to the fullerene.

\section{Reorganization energy $\lambda_{F}$}

In Fig. 7(c) we present the time evolution of the fullerene population for different values of charge-transfer reorganization energy $\lambda_{F}$. This parameter can be decreased by replacing the polar solvent with another one which has a much lower polarity. As can be seen from Fig. 7(c), the quantum

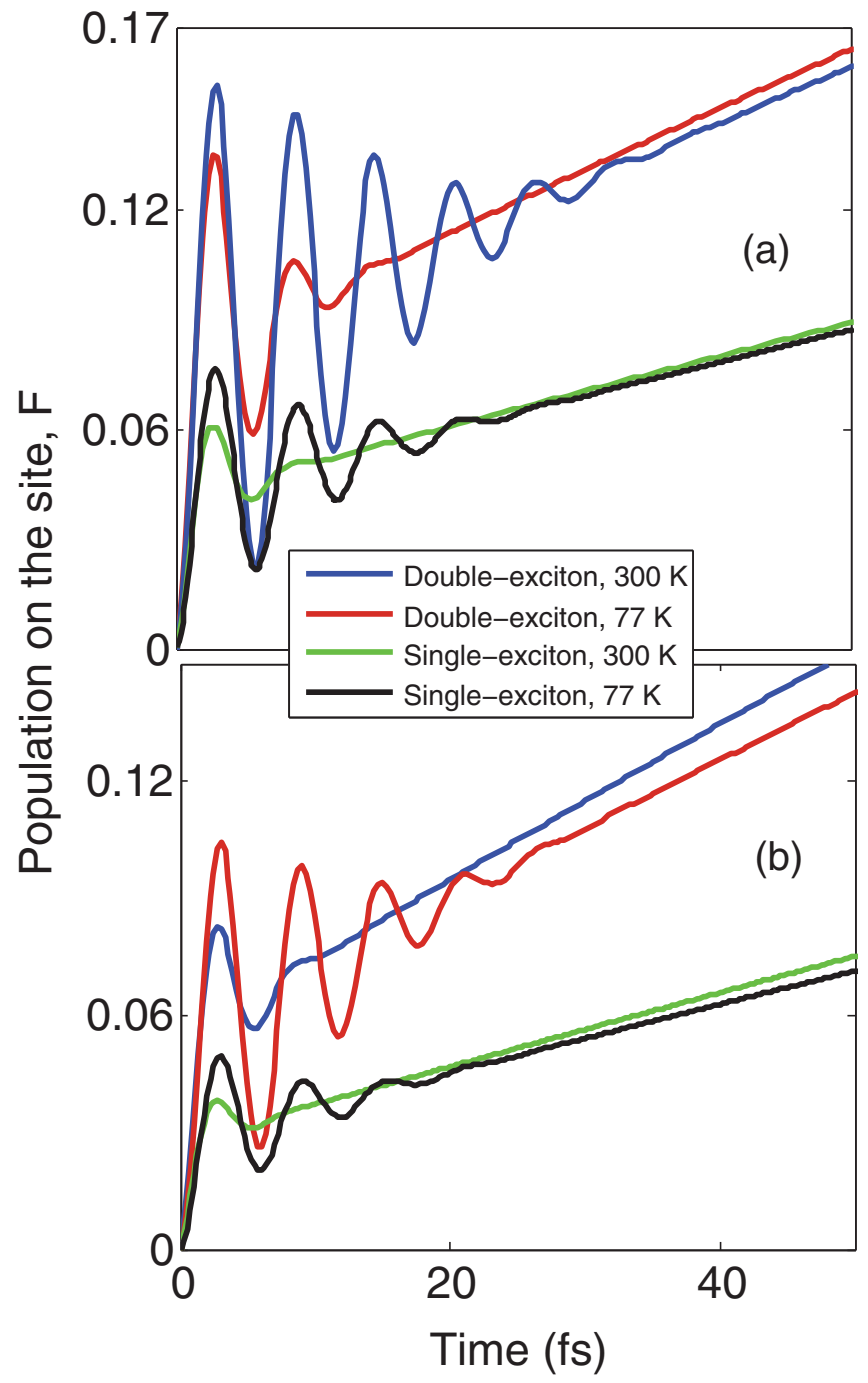

FIG. 6. Time evolution of the populations on the site F, for both sets of parameters, I and II, comparing the double-exciton case (the two ZnPy chromophores are excited) with the single-exciton case. (a) Time evolution of the populations on the site F for the parameter set I. (b) Time evolution of the populations on the site F for the parameter set II. Note that the doubleexcitation significantly enhances the amplitude of the charge oscillations at the fullerene site for both sets of parameters, either at low or at high temperatures.

oscillations of the fullerene charge survive much longer times for smaller values of the reorganization energy, which correspond to weaker system-environment couplings. A similar effect is expected when the porphyrin reorganization energy is changed.

\section{CONCLUSIONS}

We theoretically studied the energy and electron-transfer dynamics in a wheel-shaped artificial antenna-reaction center complex. This complex ${ }^{17}$ mimicking a natural photosystem, contains six chromophores $\left(\mathrm{BPEA}_{a}, \mathrm{BPEA}_{b}, \mathrm{BDPY}_{a}\right.$, $\mathrm{BDPY}_{b}, \mathrm{ZnPy}_{a}$, and $\mathrm{ZnPy}_{b}$ ) and an electron acceptor (fullerene, F). Using the methods of dissipative quantum mechanics we derive and solve a set of equations for both the diagonal and off-diagonal elements of the density matrix, which describe quantum coherent effects in energy and charge 


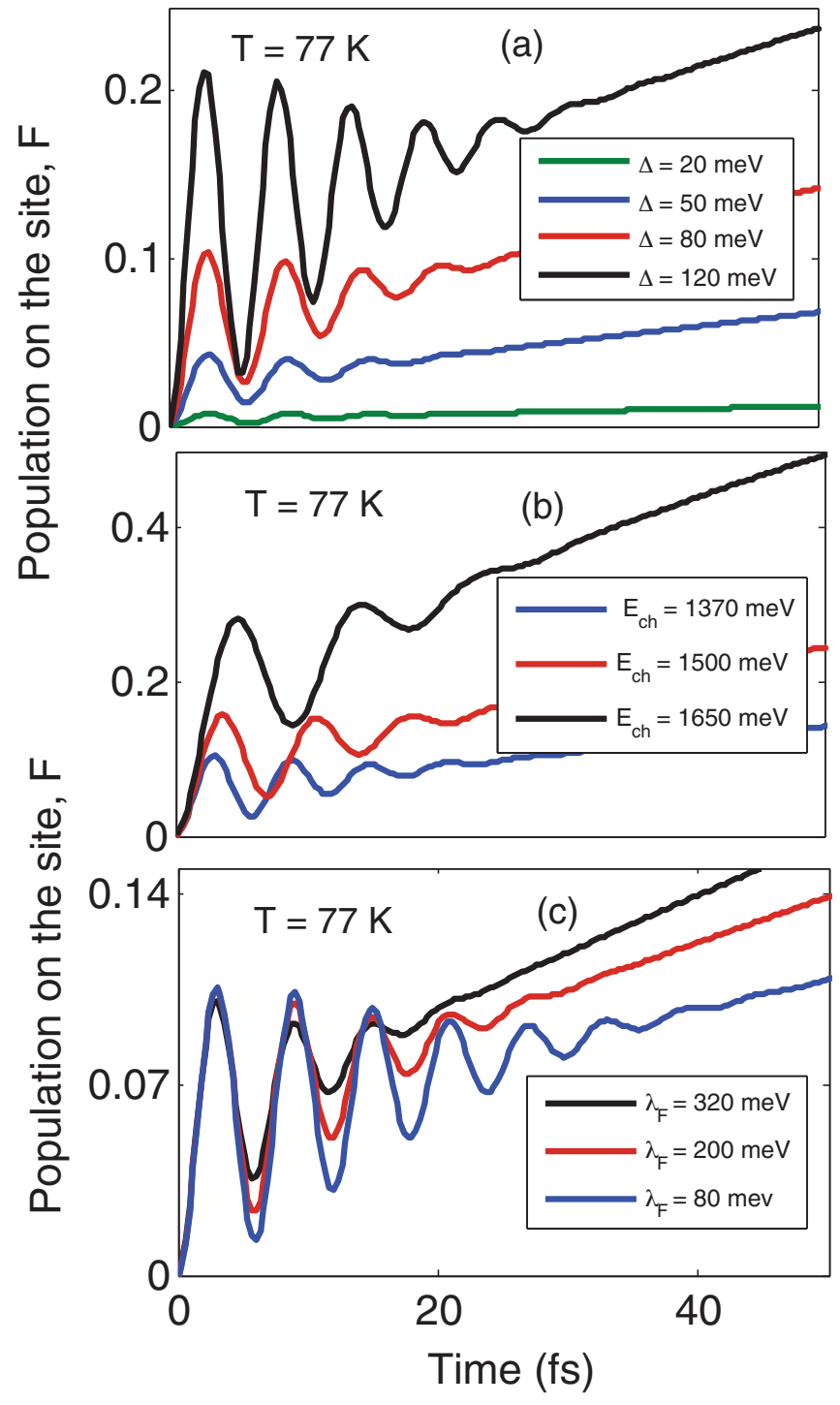

FIG. 7. Time evolution of the population on the site $\mathrm{F}$ for the parameter set II when both $\mathrm{ZnPy}$ chromophores are excited at $t=0$. (a) Effects of the coupling $\Delta$ on the time evolution of the populations on the site F. (b) Effects of the energy gap between an excited state of a $\mathrm{ZnPy}$ chromophore and the charge-separated state, $E_{\mathrm{ch}}$, on the time evolution of populations on the site $\mathrm{F}$. (c) Effects of the reorganization energy $\lambda$ on the time evolution of populations on the site F. As can be seen from these plots, the contribution of wavelike coherent motion to electron-transfer dynamics is significantly enhanced when strengthening the coupling between fullerene and porphyrin, lowering the energy gap between the fullerene and porphyrin sites, and decreasing the reorganization energy.

transfer. We consider two sets of parameters, one corresponding to the case where the energy-transfer reorganization energy $\Lambda$ is less than the resonant coupling $V$ between the chromophores, $\Lambda<V$, and another regime where $\Lambda>V$. For these two sets of parameters, we examine the electron and exciton dynamics, with special emphasis on the shorttime regime ( $\sim$ femtoseconds). We demonstrate that, in agreement with the experiments performed in Ref. 17, the excitation energy of the BPEA antenna chromophores is efficiently funneled to porphyrins ( $\mathrm{ZnPy}$ ). The excited $\mathrm{ZnPy}$ molecules rapidly donate an electron to the fullerene electron acceptor, thus creating a charge-separated state, $\mathrm{ZnPy}^{+}-\mathrm{F}^{-}$, with a quantum yield of the order of $95 \%$. There is no observable difference in energy transduction efficiency for these two sets of parameters. In the limit of strong interchromophoric coupling, coherent dynamics dominates over incoherent-hopping motion. In the single-exciton regime, when one of the BPEA chromophores is initially excited, quantum beatings between two resonant BPEA chromophores occur with decoherence times of the order of $100 \mathrm{fs}$. However, here the electron transfer process is dominated by incoherent hopping. For the case where one porphyrin molecule is excited at the beginning, we obtain small quantum oscillations of the fullerene charge characterized by a short decay time scale $(\sim 10 \mathrm{fs})$. More pronounced quantum oscillations of the fullerene charge (with an amplitude $\sim 0.1$ electron charge and decoherence time of about $20 \mathrm{fs}$ at $T=77 \mathrm{~K}$ ) are predicted for the double-exciton regime, when both porphyrin molecules are initially excited. We also show that the contribution of wave-like coherent motion to electron-transfer dynamics could be enhanced by lowering the temperature, strengthening the fullerene-porphyrin bonds, shrinking the energy gap between the zinc porphyrin and fullerene moieties (e.g., by attaching a charged residue to the fullerene), as well as by decreasing the reorganization energy (by tuning the solvent polarity).

\section{ACKNOWLEDGMENTS}

We thank the RIKEN RICC for providing computing resources. F.N. acknowledges partial support from the Laboratory of Physical Sciences, the National Security Agency, the Army Research Office, the Defense Advanced Research Projects Agency (DARPA), the Air Force Office of Scientific Research, the National Science Foundation (Grant No. 0726909), JSPS-RFBR (Contract No. 09-02-92114), the Grant-in-Aid for Scientific Research (S), MEXT Kakenhi on Quantum Cybernetics, and the Funding Program for Innovative Research and Development on Science and Technology (FIRST).

\section{APPENDIX A: COULOMB INTERACTION ENERGIES}

The Coulomb interactions between the electron states are

$$
\begin{aligned}
H_{C}= & -u_{F}\left[\left(1-\bar{n}_{\mathrm{ZnPy}_{a}}\right) n_{F}+\left(1-\bar{n}_{\mathrm{ZnPy}_{b}}\right) n_{F}\right] \\
& +u_{\mathrm{Py}}\left(1-\bar{n}_{\mathrm{ZnPy}_{a}}\right)\left(1-\bar{n}_{\mathrm{ZnPy}_{b}}\right) \\
& +u_{\mathrm{ZnPy}_{a}} n_{\mathrm{ZnPy}_{a}} n_{\mathrm{ZnPy}_{a}^{*}}+u_{\mathrm{ZnPy}_{b}} n_{\mathrm{ZnPy}_{b}} n_{\mathrm{ZnPy}_{b}^{*}},
\end{aligned}
$$

where

$\bar{n}_{\mathrm{ZnPy}_{a}}=n_{\mathrm{ZnPy}_{a}}+n_{\mathrm{ZnPy}_{a}^{*}} \quad$ and $\quad \bar{n}_{\mathrm{ZnPy}_{b}}=n_{\mathrm{ZnPy}_{b}}+n_{\mathrm{ZnPy}_{b}^{*}}$.

The first term of Eq. (A1) represents the electrostatic attraction (so the minus sign) between the positively charged $\mathrm{ZnPy}$ chromophores and the negatively charged fullerene. The second term is due to the Coulomb repulsion (so the plus sign) between two $\mathrm{ZnPy}$ chromophores. The last two terms are the repulsive interaction energies when both the excited and ground states of the $\mathrm{ZnPy}$ chromophores are occupied by electrons. The coefficients $u_{F}, u_{\mathrm{Py}}, u_{\mathrm{ZnPy}_{a}}$, and $u_{\mathrm{ZnPy}_{a}}$ represent the magnitudes of the electrostatic interactions and these are calculated using the Coulomb formula. We have assumed that the empty ZnPy chromophores $\left(n_{\mathrm{ZnPy}}+n_{\mathrm{ZnPy}^{*}}=0\right)$ have 
positive charges and the acceptor state $\mathrm{F}$ becomes negatively charged when it is occupied by an electron.

\section{APPENDIX B: DERIVATION OF EQUATIONS FOR THE MATRIX $\left\langle\rho_{\mu \nu}\right\rangle$}

Our derivation of the equations for the matrix $\left\langle\rho_{\mu \nu}\right\rangle$ is based on the exact solution for the operator $\rho_{\mu \nu}=(|\mu\rangle\langle\nu|)(t)$ of the system influenced only by diagonal fluctuations of the bath. In this case the "system + bath" Hamiltonian has the form

$$
\begin{aligned}
H_{\text {diag }}= & \sum_{\mu} E_{\mu}|\mu\rangle\langle\mu|+\sum_{j}\left(\frac{p_{j}^{2}}{2 m_{j}}+\frac{m_{j} \omega_{j}^{2} x_{j}^{2}}{2}\right) \\
& -\sum_{\mu} \sum_{j} m_{j} \omega_{j}^{2} \Lambda_{j}^{\mu} x_{j}|\mu\rangle\langle\mu|,
\end{aligned}
$$

where $\Lambda_{j}^{\mu}=\Lambda_{j}^{\mu \mu}$ [see Eq. (13)]. The time evolution of the exciton operators $\rho_{\mu \nu}$ is governed by the Heisenberg equation

$$
i \dot{\rho}_{\mu \nu}=-\omega_{\mu \nu} \rho_{\mu \nu}+\sum_{j} m_{j} \omega_{j}^{2}\left(\Lambda_{j}^{\mu}-\Lambda_{j}^{v}\right) x_{j} \rho_{\mu \nu} .
$$

It is possible to verify that the solution of Eq. (B2) is given by the equation

$$
\begin{aligned}
\rho_{\mu \nu}(t)= & \exp \left[i \Omega_{\mu \nu}\left(t-t_{0}\right)\right] \times \exp \left[i \sum_{j} p_{j}(t)\left(\Lambda_{j}^{\mu}-\Lambda_{j}^{\nu}\right)\right] \\
& \times \exp \left[-i \sum_{j} p_{j}\left(t_{0}\right)\left(\Lambda_{j}^{\mu}-\Lambda_{j}^{\nu}\right)\right] \rho_{\mu \nu}\left(t_{0}\right),
\end{aligned}
$$

where

$$
\Omega_{\mu \nu}=\omega_{\mu \nu}-\sum_{j} \frac{m_{j} \omega_{j}^{2}}{2}\left[\left(\Lambda_{j}^{\mu}\right)^{2}-\left(\Lambda_{j}^{v}\right)^{2}\right],
$$

and $p_{j}$ is the Heisenberg operator of the dissipative environment. The evolution begins at time $t=t_{0}$. The diagonal operators $\rho_{\mu}=\rho_{\mu \mu}$ are constant, $\rho_{\mu}(t)=\rho_{\mu}\left(t_{0}\right)$, in the presence of a strong interaction with the diagonal operators of the protein environment.

For uncorrelated diagonal and off-diagonal environment operators, when $\left\langle Q_{\alpha}^{(0)}(t) \widetilde{Q}_{\mu \nu}^{(0)}\left(t^{\prime}\right)\right\rangle=0$, the contribution of the environment to the non-Markovian equation (20) consists of two parts:

$$
\begin{aligned}
\left\langle-i\left[\rho_{\mu \nu}, H_{e-\mathrm{ph}}\right]_{-}\right\rangle= & \left\langle-i\left[\rho_{\mu \nu}, H_{e-\mathrm{ph}}^{\mathrm{diag}}\right]_{-}\right\rangle \\
& +\left\langle-i\left[\rho_{\mu \nu}, H_{e-\mathrm{ph}}^{\mathrm{n}-\mathrm{diag}}\right]_{-}\right\rangle .
\end{aligned}
$$

The diagonal elements, $Q_{\mu}$, of the environment contribute to the first part,

$$
\begin{aligned}
\langle- & \left.i\left[\rho_{\mu \nu}, H_{e-\mathrm{ph}}^{\mathrm{diag}}\right]_{-}\right\rangle \\
= & \int_{0}^{t} d t_{1}\left\langle\left(Q_{\mu}^{(0)}-Q_{\nu}^{(0)}\right)(t) Q_{\bar{\nu}}^{(0)}\left(t_{1}\right)\right\rangle\left\langle\rho_{\mu \nu}(t) \rho_{\bar{\nu}}\left(t_{1}\right)\right\rangle \\
& \quad-\int_{0}^{t} d t_{1}\left\langle Q_{\bar{\nu}}^{(0)}\left(t_{1}\right)\left(Q_{\mu}^{(0)}-Q_{\nu}^{(0)}\right)(t)\right\rangle\left\langle\rho_{\bar{\nu}}\left(t_{1}\right) \rho_{\mu \nu}(t)\right\rangle,
\end{aligned}
$$

whereas the second part is due to a contribution of the nondiagonal (abbreviated as n-diag in the super-index) operators, $\tilde{Q}_{\mu \nu}$,

$$
\begin{aligned}
\left\langle-i\left[\rho_{\mu \nu}, H_{e-\mathrm{ph}}^{\mathrm{n}-\mathrm{diag}}\right]_{-}\right\rangle & \\
= & -\int_{0}^{t} d t_{1}\left\langle\tilde{Q}_{\nu \alpha}^{(0)}(t) \tilde{Q}_{\bar{\mu} \bar{\nu}}^{(0)}\left(t_{1}\right)\right\rangle\left\langle\rho_{\mu \alpha}(t) \rho_{\bar{\mu} \bar{\nu}}\left(t_{1}\right)\right\rangle \\
& +\int_{0}^{t} d t_{1}\left\langle\tilde{Q}_{\bar{\mu} \bar{\nu}}^{(0)}\left(t_{1}\right) \tilde{Q}_{\nu \alpha}^{(0)}(t)\right\rangle\left\langle\rho_{\bar{\mu} \bar{\nu}}\left(t_{1}\right) \rho_{\mu \alpha}(t)\right\rangle \\
& +\int_{0}^{t} d t_{1}\left\langle\tilde{Q}_{\alpha \mu}^{(0)}(t) \tilde{Q}_{\bar{\mu} \bar{\nu}}^{(0)}\left(t_{1}\right)\right\rangle\left\langle\rho_{\alpha \nu}(t) \rho_{\bar{\mu} \bar{\nu}}\left(t_{1}\right)\right\rangle \\
& -\int_{0}^{t} d t_{1}\left\langle\tilde{Q}_{\bar{\mu} \bar{\nu}}^{(0)}\left(t_{1}\right) \tilde{Q}_{\alpha \mu}^{(0)}(t)\right\rangle\left\langle\rho_{\bar{\mu} \bar{\nu}}\left(t_{1}\right) \rho_{\alpha \nu}(t)\right\rangle .
\end{aligned}
$$

We note that the time evolution of the diagonal elements of the system operator, $\rho_{\mu}=\rho_{\mu \mu}$, is determined by the nondiagonal operators $\tilde{Q}_{\mu \nu}$ as well as by quenching terms. Strong diagonal fluctuations of the environment have no effect on the evolution of the diagonal elements of the matrix. Thus, in Eq. (B6) we assume $\rho_{\bar{v}}\left(t_{1}\right)=\rho_{\bar{v}}(t)$ so that Eq. (B6) can be rewritten as

$$
\left\langle-i\left[\rho_{\mu \nu}, H_{e-\mathrm{ph}}^{\mathrm{diag}}\right]_{-}\right\rangle=-\left(\Gamma_{\mu \nu}^{\mathrm{diag}}+i \delta \Omega_{\mu \nu}^{\mathrm{diag}}\right)(t)\left\langle\rho_{\mu \nu}(t)\right\rangle,
$$

where the time-dependent rate, $\Gamma_{\mu \nu}^{\mathrm{diag}}(t)$, and the frequency shift, $\delta \Omega_{\mu \nu}^{\mathrm{diag}}$, can be found from the following expression:

$$
\begin{aligned}
\Gamma_{\mu \nu}^{\operatorname{diag}}(t)+i \delta \Omega_{\mu \nu}^{\operatorname{diag}}(t)= & \int_{0}^{t} d t_{1}\left\{\left\langle\left(Q_{\mu}^{(0)}-Q_{\nu}^{(0)}\right)(t) Q_{\nu}^{(0)}\left(t_{1}\right)\right\rangle\right. \\
& \left.-\left\langle Q_{\mu}^{(0)}\left(t_{1}\right)\left(Q_{\mu}^{(0)}-Q_{\nu}^{(0)}\right)(t)\right\rangle\right\} .
\end{aligned}
$$

The rate $\Gamma_{\mu \nu}^{\text {diag }}(t)$ determines the fast decay of quantum coherence in our system. For an environment composed of independent oscillators, we obtain

$$
\begin{aligned}
\left\langle\left(Q_{\mu}^{(0)}-Q_{v}^{(0)}\right)(t) Q_{v}^{(0)}\left(t_{1}\right)\right\rangle-\left\langle Q_{\mu}^{(0)}\left(t_{1}\right)\left(Q_{\mu}^{(0)}-Q_{v}^{(0)}\right)(t)\right\rangle \\
=-\sum_{j} \frac{m_{j} \omega_{j}^{3}}{2}\left(\Lambda_{j}^{\mu}-\Lambda_{j}^{v}\right)^{2} \operatorname{coth}\left(\frac{\omega_{j}}{2 T}\right) \cos \omega_{j}\left(t-t_{1}\right) \\
\quad-i \sum_{j} \frac{m_{j} \omega_{j}^{3}}{2}\left[\left(\Lambda_{j}^{\mu}\right)^{2}-\left(\Lambda_{j}^{v}\right)^{2}\right] \sin \omega_{j}\left(t-t_{1}\right) . \quad(\mathrm{B} 10
\end{aligned}
$$

The fluctuations of the diagonal operators of the environment can be described by the set of spectral functions,

$$
\begin{aligned}
J_{\mu}(\omega) & =\sum_{j} \frac{m_{j} \omega_{j}^{3}}{2}\left(\Lambda_{j}^{\mu}\right)^{2} \delta\left(\omega-\omega_{j}\right), \\
\bar{J}_{\mu \nu}(\omega) & =\sum_{j} \frac{m_{j} \omega_{j}^{3}}{2}\left(\Lambda_{j}^{\mu}-\Lambda_{j}^{\nu}\right)^{2} \delta\left(\omega-\omega_{j}\right),
\end{aligned}
$$

together with the corresponding reorganization energies,

$$
\begin{aligned}
\lambda_{\mu} & =\int_{0}^{\infty} \frac{d \omega}{\omega} J_{\mu}(\omega)=\sum_{j} \frac{m_{j} \omega_{j}^{2}}{2}\left(\Lambda_{j}^{\mu}\right)^{2}, \\
\bar{\lambda}_{\mu \nu} & =\int_{0}^{\infty} \frac{d \omega}{\omega} \bar{J}_{\mu \nu}(\omega)=\sum_{j} \frac{m_{j} \omega_{j}^{2}}{2}\left(\Lambda_{j}^{\mu}-\Lambda_{j}^{v}\right)^{2} .
\end{aligned}
$$


We also introduce a spectral function, $\tilde{J}_{\mu \nu}(\omega)$, which characterizes the non-diagonal $(\mu \neq v)$ environment fluctuations,

$$
\tilde{J}_{\mu \nu}(\omega)=\sum_{j} \frac{m_{j} \omega_{j}^{3}}{2}\left|\tilde{\Lambda}_{j}^{\mu \nu}\right|^{2} \delta\left(\omega-\omega_{j}\right),
$$

where $\tilde{\Lambda}_{j}^{\mu \nu}=\Lambda_{j}^{\mu \nu}$ [see Eq. (13)] taken at $\mu \neq \nu$. With Eq. (B10) we calculate the contributions of the diagonal environment fluctuations into the decoherence rate and the frequency shift of the off-diagonal elements of the system matrix $\left\langle\rho_{\mu \nu}\right\rangle$ in Eq. (B8),

$$
\begin{aligned}
\Gamma_{\mu \nu}^{\mathrm{diag}}(t) & =\int_{0}^{\infty} \frac{d \omega}{\omega} \bar{J}_{\mu \nu}(\omega) \operatorname{coth}\left(\frac{\omega}{2 T}\right) \sin \omega t, \\
\delta \Omega_{\mu \nu}^{\mathrm{diag}}(t) & =\int_{0}^{\infty} \frac{d \omega}{\omega}\left[J_{\mu}(\omega)-J_{\nu}(\omega)\right](1-\cos \omega t) .
\end{aligned}
$$

The contribution of the non-diagonal fluctuations of the environment to the evolution of the electron operators $\left\langle\rho_{\mu \nu}\right\rangle$ is defined by Eq. (B7). To calculate the products of exciton variables taken at different moments of time, for example, $\rho_{\mu \alpha}(t) \rho_{\bar{\mu} \bar{\nu}}\left(t_{1}\right)$, we use Eq. (B3), which describes the evolution of exciton operators in the presence of strong coupling to the diagonal operators, $Q_{\mu}$, of the environment. We assume that the interaction with the non-diagonal environment operators, $\tilde{Q}_{\mu \nu}$, is weak. With Eq. (B3) we express the operators at time $t_{1}$ in terms of operators taken at time $t$ :

$$
\begin{aligned}
\rho_{\bar{\mu} \bar{\nu}}\left(t_{1}\right)= & \exp \left[-i \Omega_{\bar{\mu} \bar{\nu}} \tau\right] \\
& \times \exp \left[i u_{\bar{\mu} \bar{\nu}}(\tau)\right] \exp \left[-i v_{\bar{\mu} \bar{\nu}}\left(t, t_{1}\right)\right] \rho_{\mu \nu}(t), \rho_{\bar{\mu} \bar{\nu}}\left(t_{1}\right) \\
= & \rho_{\mu \nu}(t) \exp \left[-i \Omega_{\bar{\mu} \bar{\nu}} \tau\right] \exp \left[-i u_{\bar{\mu} \bar{\nu}}(\tau)\right] \\
& \times \exp \left[-i v_{\bar{\mu} \bar{\nu}}\left(t, t_{1}\right)\right],
\end{aligned}
$$

where $\tau=t-t_{1}$, and

$$
\begin{aligned}
u_{\mu \nu}(\tau) & =\int_{0}^{\infty} \frac{d \omega}{\omega} \bar{J}_{\mu \nu}(\omega) \sin \omega \tau, \\
v_{\mu \nu}\left(t, t_{1}\right) & =\sum_{j}\left(\Lambda_{j}^{\mu}-\Lambda_{j}^{\nu}\right)\left[p_{j}(t)-p_{j}\left(t_{1}\right)\right] .
\end{aligned}
$$

Here, we assume that $p_{j}(t), p_{j}\left(t_{1}\right)$ are free-evolving momentum operators of the environment, which are described by Gaussian statistics with a correlation function

$$
\left\langle\frac{1}{2}\left[p_{j}(t), p_{j}\left(t_{1}\right)\right]_{+}\right\rangle=\frac{\hbar m_{j} \omega_{j}}{2} \operatorname{coth}\left(\frac{\hbar \omega_{j}}{2 T}\right) \cos \omega_{j}\left(t-t_{1}\right) \text {. }
$$

The operator function $v_{\mu \nu}\left(t, t_{1}\right)$ does not commute with the exciton matrix $\rho_{\mu \nu}(t)$, and, therefore, we need two expressions for the operator $\rho_{\mu \nu}\left(t_{1}\right)$, which are distinguished by the order of the operators $\rho_{\mu \nu}(t)$ and $\exp \left[-i v_{\mu \nu}\left(t, t_{1}\right)\right]$. For the average value of the operator $\exp \left[-i v_{\mu \nu}\left(t, t_{1}\right)\right]$, we obtain

$$
\begin{aligned}
& \left\langle\exp \left[-i v_{\mu \nu}\left(t, t_{1}\right)\right]\right\rangle \\
& =\exp \left\{-\int_{0}^{\infty} \frac{d \omega}{\omega^{2}} \bar{J}_{\mu \nu}(\omega) \operatorname{coth}\left(\frac{\hbar \omega}{2 T}\right)\left[1-\cos \omega\left(t-t_{1}\right)\right]\right\} .
\end{aligned}
$$

Substituting Eq. (B15) into Eq. (B7) and using the secular approximation, we obtain a contribution of the non-diagonal environment operators, $\tilde{Q}_{\mu \nu}$, to the evolution of diagonal exciton operators $\left\langle\rho_{\mu}\right\rangle$,

$$
\left\langle-i\left[\rho_{\mu}, H_{e \text {-ph }}^{\mathrm{n} \text {-diag }}\right]_{-}\right\rangle=-\sum_{\alpha} \tilde{\gamma}_{\alpha \mu}(t)\left\langle\rho_{\mu}\right\rangle+\sum_{\alpha} \tilde{\gamma}_{\mu \alpha}(t)\left\langle\rho_{\alpha}\right\rangle,
$$

characterized by the following relaxation matrix:

$$
\begin{aligned}
& \tilde{\gamma}_{\mu \alpha}(t) \\
& =\int_{0}^{t} d t_{1}\left\langle\tilde{Q}_{\alpha \mu}^{(0)}(t) \tilde{Q}_{\mu \alpha}^{(0)}\left(t_{1}\right)\right\rangle e^{-i \Omega_{\mu \alpha}\left(t-t_{1}\right)} e^{-i u_{\mu \alpha}\left(t-t_{1}\right)}\left\langle e^{-i v_{\mu \alpha}\left(t, t_{1}\right)}\right\rangle \\
& \quad+\int_{0}^{t} d t_{1}\left\langle\tilde{Q}_{\alpha \mu}^{(0)}\left(t_{1}\right) \tilde{Q}_{\mu \alpha}^{(0)}(t)\right\rangle e^{-i \Omega_{\alpha \mu}\left(t-t_{1}\right)} e^{i u_{\alpha \mu}\left(t-t_{1}\right)}\left\langle e^{-i v_{\alpha \mu}\left(t, t_{1}\right)}\right\rangle,
\end{aligned}
$$

where

$$
\begin{aligned}
\left\langle\tilde{Q}_{\alpha \mu}^{(0)}(t) \tilde{Q}_{\mu \alpha}^{(0)}\left(t_{1}\right)\right\rangle= & (1 / 2) \int_{0}^{\infty} \tilde{J}_{\alpha \mu}(\omega) \\
& \times\left\{\left[\operatorname{coth}\left(\frac{\omega}{2 T}\right)-1\right] e^{i \omega\left(t-t_{1}\right)}\right. \\
& \left.+\left[\operatorname{coth}\left(\frac{\omega}{2 T}\right)+1\right] e^{-i \omega\left(t-t_{1}\right)}\right\} .
\end{aligned}
$$

When the environment is at high temperatures $(2 T \gg \omega)$ and at low frequencies of the diagonal fluctuations $(\omega \tau \ll 1)$, we have

$$
u_{\mu \nu}(\tau) \simeq \bar{\lambda}_{\mu \nu} \tau,
$$

and

$$
\left\langle\exp \left[-i v_{\mu \nu}\left(t, t_{1}\right)\right]\right\rangle \simeq \exp \left[-\bar{\lambda}_{\mu \nu} T\left(t-t_{1}\right)^{2}\right] .
$$

With these assumptions the relaxation matrix has a simple form

$$
\begin{aligned}
\tilde{\gamma}_{\mu \alpha}= & \sqrt{\frac{\pi}{\bar{\lambda}_{\alpha \mu}}} \int_{0}^{\infty} d \omega \tilde{J}_{\alpha \mu}(\omega) n(\omega) \\
& \times\left\{\exp \left[-\frac{\left(\omega+\Omega_{\alpha \mu}-\bar{\lambda}_{\alpha \mu}\right)^{2}}{4 \bar{\lambda}_{\alpha \mu} T}\right]\right. \\
& \left.+\exp \left(\frac{\omega}{T}\right) \exp \left[-\frac{\left(\omega-\Omega_{\alpha \mu}+\bar{\lambda}_{\alpha \mu}\right)^{2}}{4 \bar{\lambda}_{\alpha \mu} T}\right]\right\},
\end{aligned}
$$

where $n(\omega)=[\exp (\omega / T)-1]^{-1}$ is the Bose distribution function at the temperature $T$. The moment of time $t$ in the expression (B20) for the relaxation matrix is usually higher than the effective retardation time, $\tau_{c} \sim\left(\bar{\lambda}_{\alpha \mu} T\right)^{-1 / 2}$, of the integrand in Eq. (B20): $t \gg \tau_{c}$. Therefore, we assume that $t \simeq \infty$ so that $\tilde{\gamma}_{\mu \alpha}(t) \simeq \tilde{\gamma}_{\mu \alpha}(\infty)=\tilde{\gamma}_{\mu \alpha}$.

It follows from Eq. (B7) that a contribution of the nondiagonal environment operators $\tilde{Q}_{\mu \nu}$ to the evolution of the off-diagonal elements $\rho_{\mu \nu}$ is given by the formula

$$
\left\langle-i\left[\rho_{\mu \nu}, H_{e-\mathrm{ph}}^{\mathrm{n} \text {-diag }}\right]_{-}\right\rangle=-\left(\tilde{\Gamma}_{\mu \nu}+i \delta \tilde{\Omega}_{\mu \nu}\right)(t)\left\langle\rho_{\mu \nu}(t)\right\rangle,
$$


where

$$
\begin{aligned}
& \tilde{\Gamma}_{\mu \nu}(t)+i \delta \tilde{\Omega}_{\mu \nu}(t) \\
& =\int_{0}^{t} d t_{1}\left\langle\tilde{Q}_{\nu \alpha}^{(0)}(t) \tilde{Q}_{\alpha \nu}^{(0)}\left(t_{1}\right)\right\rangle e^{-i \Omega_{\alpha \nu}\left(t-t_{1}\right)} e^{-i u_{\alpha \nu}\left(t-t_{1}\right)}\left\langle e^{-i v_{\alpha \nu}\left(t, t_{1}\right)}\right\rangle \\
& \quad+\int_{0}^{t} d t_{1}\left|\tilde{Q}_{\mu \alpha}^{(0)}(t) \tilde{Q}_{\alpha \mu}^{(0)}\left(t_{1}\right)\right\rangle e^{-i \Omega_{\mu \alpha}\left(t-t_{1}\right)} e^{i u_{\mu \alpha}\left(t-t_{1}\right)}\left\langle e^{-i v_{\mu \alpha}\left(t, t_{1}\right)}\right\rangle .
\end{aligned}
$$

A small frequency shift, $\delta \tilde{\Omega}_{\mu \nu}$, can be hereafter ignored. The dephasing rate, $\tilde{\Gamma}_{\mu \nu}$, has two parts, $\tilde{\Gamma}_{\mu \nu}=\tilde{\Gamma}_{\mu}+\tilde{\Gamma}_{\nu}$, where

$$
\begin{aligned}
\tilde{\Gamma}_{\mu}= & \frac{1}{2} \sum_{\alpha} \sqrt{\frac{\pi}{\bar{\lambda}_{\mu \alpha} T}} \int_{0}^{\infty} d \omega \tilde{J}_{\mu \alpha}(\omega) n(\omega) \\
& \times\left\{\exp \left[-\frac{\left(\omega+\Omega_{\mu \alpha}-\bar{\lambda}_{\mu \alpha}\right)^{2}}{4 \bar{\lambda}_{\mu \alpha} T}\right]\right. \\
& \left.+\exp \left(\frac{\omega}{T}\right) \exp \left[-\frac{\left(\omega-\Omega_{\mu \alpha}+\bar{\lambda}_{\mu \alpha}\right)^{2}}{4 \bar{\lambda}_{\mu \alpha} T}\right]\right\} .
\end{aligned}
$$

We note that $\tilde{\Gamma}_{\mu}=(1 / 2) \sum_{\alpha} \tilde{\gamma}_{\alpha \mu}$, and $\Omega_{\mu \nu}=\omega_{\mu \nu}-\lambda_{\mu}+\lambda_{v}$ from Eqs. (B4) and (B12).

Assuming that the environment fluctuations acting on each electron-binding site are independent and using Eq. (13) for the coefficients $\Lambda_{j}^{\mu \nu}$, we obtain

$$
\begin{aligned}
\tilde{J}_{\mu \nu}(\omega)= & \sum_{l}\left[J_{l S}(\omega)\left|\left\langle\mu\left|S_{l}\right| \nu\right\rangle\right|^{2}+J_{l M}(\omega)\left|\left\langle\mu\left|M_{l}\right| \nu\right\rangle\right|^{2}\right] \\
& +J_{F}(\omega)\left|\left\langle\mu\left|n_{F}\right| \nu\right\rangle\right|^{2}
\end{aligned}
$$

where

$$
\begin{aligned}
& J_{l S}(\omega)=\sum_{j} \frac{m_{j} \omega_{j}^{3}}{2} \bar{x}_{j l}^{2} \delta\left(\omega-\omega_{j}\right), \\
& J_{l M}(\omega)=\sum_{j} \frac{m_{j} \omega_{j}^{3}}{2} \tilde{x}_{j l}^{2} \delta\left(\omega-\omega_{j}\right), \\
& J_{F}(\omega)=\sum_{j} \frac{m_{j} \omega_{j}^{3}}{2} x_{j F}^{2} \delta\left(\omega-\omega_{j}\right) .
\end{aligned}
$$

The results obtained above are valid for an arbitrary frequency dependence of the spectral densities $J_{l S}(\omega), J_{l M}(\omega)$, and $J_{F}(\omega)$. Hereafter we assume that these functions are described by the Lorentz-Drude formula characterized by a common inverse correlation time, $\gamma_{c}=\tau_{c}^{-1}$, and by a corresponding reorganization energy $\lambda_{l S}, \lambda_{l M}$, or $\lambda_{F}$, e.g.,

$$
J_{l S}(\omega)=2 \frac{\lambda_{l S}}{\pi} \frac{\omega \gamma_{c}}{\omega^{2}+\gamma_{c}^{2}}
$$

Quenching processes also contribute to the decay of the off-diagonal elements, $\left\langle\rho_{\mu \nu}\right\rangle$, with the following decoherence rates: $\Gamma_{\mu \nu}^{\text {quen }}=\Gamma_{\mu}^{\text {quen }}+\Gamma_{\nu}^{\text {quen }}$, where

$$
\Gamma_{\mu}^{\text {quen }}=\sum_{l \alpha}\left|\left\langle\mu\left|a_{l}^{\dagger} a_{l^{*}}\right| \alpha\right\rangle\right|^{2} \chi_{l}^{\prime \prime}\left(\omega_{\mu \alpha}\right)\left[\operatorname{coth}\left(\frac{\omega_{\mu \alpha}}{2 T}\right)+1\right] \text {. }
$$

Here, we consider an Ohmic quenching heat bath with the spectral density $\chi_{l}^{\prime \prime}(\omega)=\alpha_{l} \omega$, which is determined by a set of site-dependent dimensionless coupling constants $\alpha_{l} \ll 1$. The contribution of quenching to the relaxation of the diagonal elements of the electron matrix, $\left\langle\rho_{\mu}\right\rangle$, is determined by the standard Redfield term

$$
\begin{aligned}
\gamma_{\mu \nu}^{\text {quen }}= & \sum_{l}\left(\left|\left\langle\mu\left|a_{l}^{\dagger} a_{l^{*}}\right| \nu\right\rangle\right|^{2}+\left|\left\langle\nu\left|a_{l}^{\dagger} a_{l^{*}}\right| \mu\right\rangle\right|^{2}\right) \chi_{l}^{\prime \prime}\left(\omega_{\mu \nu}\right) \\
& \times\left[\operatorname{coth}\left(\frac{\omega_{\mu \nu}}{2 T}\right)-1\right] .
\end{aligned}
$$

As a result, we find that the time evolution of the offdiagonal elements of the electron matrix is determined by the expression

$$
\left\langle\rho_{\mu \nu}\right\rangle(t)=\exp \left(i \omega_{\mu \nu} t-\bar{\lambda}_{\mu \nu} T t^{2}\right) \times \exp \left(-\Gamma_{\mu \nu} t\right) \rho_{\mu \nu}(0),
$$

with the decoherence rates $\Gamma_{\mu \nu}=\Gamma_{\mu}+\Gamma_{\nu}$, where the coefficient $\Gamma_{\mu}$ contains contributions of the off-diagonal fluctuations of the environment (Eq. (B25)) as well as quenching processes $\Gamma_{\mu}^{\text {quen }}$ (Eq. (B29)): $\Gamma_{\mu}=\tilde{\Gamma}_{\mu}+\Gamma_{\mu}^{\text {quen }}$. The evolution starts at the moment $t=0$ with the initial matrix $\rho_{\mu \nu}(0)$. An effect of diagonal environment fluctuations is determined by the rate $\sqrt{\bar{\lambda}_{\mu \nu} T}$, where $\bar{\lambda}_{\mu \nu}$ is the reorganization energy defined by Eq. (B12) and $T$ is the temperature of the environment.

${ }^{1}$ R. E. Blankenship, Molecular Mechanisms of Photosynthesis (Blackwell Science, Oxford, UK, 2002).

${ }^{2}$ H. V. Amerongen, L. Valkunas, and R. van Grondelle, Photosynthetic Excitons (World Scientific, Singapore, 2000).

${ }^{3}$ G. S. Engel, T. R. Calhoun, E. L. Read, T. K. Ahn, T. Mančal, Y. C. Cheng, R. E. Blankenship, and G. R. Fleming, Nature (London) 446, 782 (2007).

${ }^{4}$ G. Panitchayangkoon, D. Hayes, K. A. Fransted, J. R. Caram, E. Harel, J. Wen, R. E. Blankenship, and G. S. Engel, Proc. Natl. Acad. Sci. U.S.A. 107, 12766 (2010).

${ }^{5}$ P. Rebentrost, M. Mohseni, I. Kassal, S. Lloyd, and A. Aspuru-Guzik, New J. Phys. 11, 033003 (2009); P. Rebentrost, R. Chakraborty, and A. AspuruGuzik, J. Chem. Phys. 131, 184102 (2009).

${ }^{6}$ M. B. Plenio and S. F. Huelga, New J. Phys. 10, 113019 (2008).

${ }^{7}$ A. Ishizaki and G. R. Fleming, Proc. Natl. Acad. Sci. U.S.A. 106, 17255 (2009).

${ }^{8}$ Y.-C. Cheng and G. R. Fleming, Annu. Rev. Phys. Chem. 60, 241 (2009).

${ }^{9}$ M. Sarovar, A. Ishizaki, G. R. Fleming, and K. B. Whaley, Nat. Phys. 6, 462 (2010).

${ }^{10}$ E. Collini and G. D. Scholes, Science 323, 369 (2009).

${ }^{11}$ J. Barber, Chem. Soc. Rev. 38, 185 (2009).

${ }^{12}$ A. W.D. Larkum, Curr. Opin. Biotechnol. 21, 271 (2010).

${ }^{13}$ G. Steinberg-Yfrach, P. A. Liddell, S. C. Hung, A. L. Moore, D. Gust, and T. A. Moore, Nature (London) 385, 239 (1997).

${ }^{14}$ G. Steinberg-Yfrach, J. L. Rigaud, E. N. Durantini, A. L. Moore, D. Gust, and T. A. Moore, Nature (London) 392, 479 (1998).

${ }^{15}$ G. Kodis, Y. Terazono, P. A. Liddell, J. Andréasson, V. Garg, H. Hambourger, T. A. Moore, A. L. Moore, and D. Gust, J. Am. Chem. Soc. 128, 1818 (2006).

${ }^{16}$ D. Gust, T. A. Moore, and A. L. Moore, Acc. Chem. Res. 42, 1890 (2009).

${ }^{17}$ Y. Terazono, G. Kodis, P. A. Liddell, V. Garg, T. A. Moore, A. L. Moore, and D. Gust, J. Phys. Chem. B 113, 7147 (2009). 
${ }^{18}$ H. Imahori, H. Yamada, Y. Nishimura, I. Yamazaki, and Y. Sakata, J. Phys. Chem. B 104, 2099 (2000).

${ }^{19}$ P. K. Ghosh, A. Yu. Smirnov, and F. Nori, J. Chem. Phys. 131, 035102 (2009).

${ }^{20}$ A. Yu. Smirnov, L. G. Mourokh, P. K. Ghosh, and F. Nori, J. Phys. Chem. C 113, 21218 (2009).

${ }^{21}$ A. J. Leggett, S. Chakravarty, A. T. Dorsey, M. P. A. Fisher, A. Garg, and W. Zwerger, Rev. Mod. Phys. 59, 1 (1987).

${ }^{22}$ A. Yu. Smirnov, L. G. Mourokh, and F. Nori, J. Chem. Phys. 130, 235105 (2009).
${ }^{23}$ Y. Tanimura, J. Phys. Soc. Jpn. 75, 082001 (2006).

${ }^{24}$ G. F. Efremov and A. Yu. Smirnov, Sov. Phys. JETP 53, 547 (1981); G.

F. Efremov, L. G. Mourokh, and A. Yu. Smirnov, Phys. Lett. A 175, 89 (1993); A. Yu. Smirnov, Phys. Rev. B 68, 134514 (2003).

${ }^{25}$ A. Ishizaki and G. R. Fleming, J. Chem. Phys. 130, 234111 (2009).

${ }^{26}$ W. M. Zhang, T. Meier, V. Chernyak, and S. Mukamel, J. Chem. Phys. 108, 7763 (1998)

${ }^{27}$ M. Yang and G. R. Fleming, Chem. Phys. 282, 163 (2002).

${ }^{28}$ Y. Terazono, G. Kodis, P. A. Liddell, V. Garg, M. Gervaldo, T. A. Moore, A. L. Moore, and G. Gust, Photochem. Photobiol. 83, 464-469 (2007). 